TRANSACTIONS OF THE

AMERICAN MATHEMATICAL SOCIETY

Volume 353, Number 5, Pages 1937-1957

S 0002-9947(00)02689-1

Article electronically published on November 29, 2000

\title{
ESSENTIAL COHOMOLOGY AND EXTRASPECIAL $p$-GROUPS
}

\author{
PHAM ANH MINH
}

\begin{abstract}
Let $p$ be an odd prime number and let $G$ be an extraspecial $p$ group. The purpose of the paper is to show that $G$ has no non-zero essential mod- $p$ cohomology (and in fact that $H^{*}\left(G, \mathbb{F}_{p}\right)$ is Cohen-Macaulay) if and only if $|G|=27$ and $\exp (G)=3$.
\end{abstract}

\section{INTRODUCTION}

Let $p$ be a prime number. For every $p$-group $K$, denote by $H^{*}(K)$ the mod$p$ cohomology ring of $K$. A mod- $p$ cohomology class of $K$ is called essential if it vanishes on restriction to every proper subgroup of $K$. Let $\operatorname{Ess}(K)$ be the ideal of $H^{*}(K)$ consisting of such classes of $H^{*}(K)$. As observed in [3], the study of $\operatorname{Ess}(K)$ could provide interesting information for $H^{*}(K)$ (but, in contrast, it seems in general rather difficult to obtain elements of $\operatorname{Ess}(K))$. For instance, $\operatorname{Ess}(K) \neq\{0\}$ implies that the depth of $H^{*}(K)$ is just the rank of the center of $K$ (see [3] and [5]); furthermore, with the condition that $H^{*}(K)$ is Cohen-Macaulay, it follows from [1] that $\operatorname{Ess}(K) \neq\{0\}$ if and only if every element of order $p$ of $K$ is central (a way to obtain some element of $\operatorname{Ess}(K)$ in this case was shown there).

We are now interested in extraspecial $p$-groups $G$. For $p=2$, it was proved by Quillen ([17]) that $H^{*}(G)$ is Cohen-Macaulay and $\operatorname{Ess}(G)=\{0\}$, except for the case $G=Q_{8}$, the quaternion group of order 8 (this fact also follows from Adem and Karagueuzian's result, as $Q_{8}$ is the unique group in which every element of order 2 is central). However, the situation is quite different for the case $p>2-$ which is assumed from now on. Consider first the case $|G|=p^{3}$; it follows from [8], [9], [10], [16] that $\operatorname{Ess}(G) \neq\{0\}$ (so $H^{*}(G)$ is not Cohen-Macaulay) if and only if $\exp (G)>3$. In order to generalize this fact, in this note, we prove

Theorem. If $G$ is an extraspecial p-group, then $\operatorname{Ess}(G)=\{0\}$ iff $\exp (G)=3$ and $|G|=3^{3}$.

It follows that the unique extraspecial $p$-group which has no non-zero essential cohomology is the one of order 27 and of exponent 3. In each of the remaining cases, $H^{*}(G)$ is not Cohen-Macaulay and the depth of $H^{*}(G)$ is just 1; we also point out some non-zero essential classes of $G$ (it turns out that, if $|G|=p^{5}$ or $\exp (G)=p^{2}$,

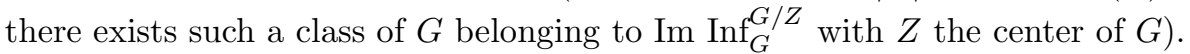

The note is organized as follows. In Section 2, given an extraspecial $p$-group $G$ of order $p^{2 n+1}$, we shall consider $G$ as a subgroup of the central product $\Gamma_{n}=C_{p^{2}} \bullet G$ and give a sufficient and necessary condition for the fact that $\operatorname{Res}_{G}^{\Gamma_{n}}(\xi) \neq 0$ with

Received by the editors September 10, 1999.

2000 Mathematics Subject Classification. Primary 20J06; Secondary 20D15, 55R40.

(C)2000 American Mathematical Society 
$\xi \in H^{*}\left(\Gamma_{n}\right)$. The proofs of the theorem for the cases $\exp (G)>p$ or $|G|=p^{5}$, which are rather simple, will be given in Section 3. Section 4 is devoted to the case $\exp (G)=p$.

\section{The GROUP $\Gamma_{n}$}

Let us recall that an extraspecial $p$-group $G$ is of order $p^{2 n+1}(n \in \mathbb{N})$ and is isomorphic to one of the following central products of groups:

$$
\begin{aligned}
\mathbb{E}_{n} & =\mathbb{E} \bullet \cdots \bullet \mathbb{E}(n \text { times }), \\
\mathbb{M}_{n} & =\mathbb{M} \bullet \mathbb{E}_{n-1},
\end{aligned}
$$

where

$$
\begin{aligned}
\mathbb{M} & =\left\langle a, b \mid a^{p^{2}}=b^{p}=1, b^{-1} a b=a^{1+p}\right\rangle, \\
\mathbb{E} & =\left\langle a, b \mid a^{p}=b^{p}=[a, b]^{p}=[a,[a, b]]=[b,[a, b]]=1\right\rangle
\end{aligned}
$$

are extraspecial $p$-groups of order $p^{3}$. Note that

$$
\exp (G)= \begin{cases}p^{2}, & \text { for } G=\mathbb{M}_{n}, \\ p, & \text { for } G=\mathbb{E}_{n},\end{cases}
$$

and $\mathbb{M}_{n}=\mathbb{M}_{n-1} \bullet \mathbb{M}$.

These groups can be obtained cohomologically as follows. Let $V$ be a vector space of dimension $2 n+1$ over the prime field $\mathbb{F}_{p}$ with basis $e, a_{1}, \ldots, a_{2 n}$. Let $x, x_{1}, \ldots, x_{2 n}$ be a basis of $H^{1}(V)$, dual to that of $V$, and let $y=\beta x, y_{i}=\beta x_{i}$ with $\beta$ the Bockstein homomorphism, so

$$
H=H^{*}(V)=E\left[x, x_{1}, \ldots, x_{2 n}\right] \otimes \mathbb{F}_{p}\left[y, y_{1}, \ldots, y_{2 n}\right]
$$

with $E[u, v, \ldots]$ (resp. $\mathbb{F}_{p}[u, v, \ldots]$ ) the exterior (resp. polynomial) algebra over $\mathbb{F}_{p}$ with generators $u, v, \ldots$ of degree 1 (resp. 2). Consider the central extension

$$
0 \rightarrow \mathbb{F}_{p} \stackrel{i}{\rightarrow} \Gamma_{n} \rightarrow V \rightarrow 0
$$

with factor set $z=z_{n}=y+x_{1} x_{2}+\cdots+x_{2 n-1} x_{2 n}$. Via the inflation map, $x$ and the $x_{i}$ 's can be considered as elements of $H^{1}\left(\Gamma_{n}\right)$. Given a subgroup $K$ of $\Gamma_{n}$, with some abuse of notation, we also denote by $x$ (resp. $x_{i}$ ) the element $\operatorname{Res}_{K}^{\Gamma_{n}}(x)$ (resp. $\left.\operatorname{Res}_{K}^{\Gamma_{n}}\left(x_{i}\right)\right)$.

It is easy to show

Lemma 1. (i) $\Gamma_{n}=C_{p^{2}} \bullet \mathbb{M}_{n}=C_{p^{2}} \bullet \mathbb{E}_{n}=\Gamma_{n-1} \bullet \mathbb{M}$.

(ii) $\mathbb{M}_{n}=\operatorname{Ker}(x+\alpha), \mathbb{E}_{n}=\operatorname{Ker} x$ and $\Gamma_{n-1} \times C_{p}=\operatorname{Ker} \alpha$, with $\alpha$ a non-zero linear combination of $x_{1}, \ldots, x_{2 n}$.

Then $C_{p^{2}}=\bigcap_{i=1}^{2 n} \operatorname{Ker} x_{i}$ is a subgroup of $\Gamma_{n}$. Let $w$ be a generator of $H^{2}\left(C_{p^{2}}\right)$, so

$$
H^{*}\left(C_{p^{2}}\right)=E[x] \otimes \mathbb{F}_{p}[w] .
$$

Set $\mathcal{G}_{n}=C_{p^{2}} \times \mathbb{E}_{n}$. By the Künneth formula, we have

$$
H^{*}\left(\mathcal{G}_{n}\right)=H^{*}\left(\mathbb{E}_{n}\right) \otimes E[x] \otimes \mathbb{F}_{p}[w] .
$$

As $\Gamma_{n}$ is the central product of $C_{p^{2}}$ and $\mathbb{E}_{n}$, there exists a central subgroup $U_{n}$ of order $p$ of $\mathcal{G}_{n}$ such that $\mathcal{G}_{n} / U_{n}=\Gamma_{n}$ and the factor set of the central extension

$$
1 \rightarrow U_{n} \rightarrow \mathcal{G}_{n} \rightarrow \Gamma_{n} \rightarrow 1
$$


is just $y$. Consider the following commutative diagram:

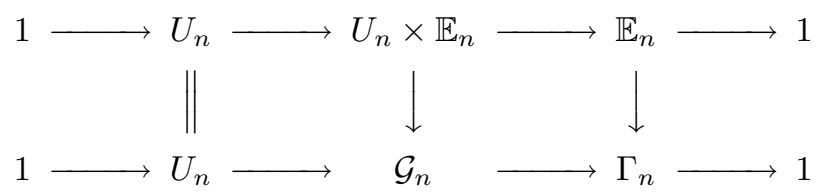

whose rows are central extensions and whose vertical arrows are inclusion maps. Pick elements $s, t$ of $H^{*}\left(U_{n}\right)$ satisfying $H^{*}\left(U_{n}\right)=E[s] \otimes P[t]$. It follows from [11] (see also 2]) that $t$ can be chosen so that $\operatorname{Res}_{U_{n}}^{\mathcal{G}_{n}}(w \times 1)=t$.

We now use the following notation. Given a ring $R$ and elements $r, s, \cdots \in R$, $(r, s, \ldots)$ will denote the ideal of $R$ generated by $r, s, \ldots$ The main result of this section is the following.

Proposition 1. If $\xi \in H^{*}\left(\Gamma_{n}\right)$, then $\operatorname{Res}_{\mathbb{E}_{n}}^{\Gamma_{n}}(\xi) \neq 0$ iff $x \xi \notin(y)$.

Proof. Set $X=\operatorname{Inf}_{\mathcal{G}_{n}}^{\Gamma_{n}}(\xi)$. As $\operatorname{Ker} \operatorname{Inf}_{\mathcal{G}_{n}}^{\Gamma_{n}}=(y)$, it follows that $x X=0$ iff $x \xi \in(y)$. Write $X=\sum w^{i} \otimes s_{i}+\sum w^{i} x \otimes t_{i}$ with $s_{i}, t_{i} \in H^{*}\left(\mathbb{E}_{n}\right)$. It is clear that $\operatorname{Res}_{\mathbb{E}_{n}}^{\Gamma_{n}}(\xi) \neq 0$ iff $\operatorname{Inf}_{U_{n} \times \mathbb{E}_{n}}^{\mathbb{E}_{n}} \operatorname{Res}_{\mathbb{E}_{n}}^{\Gamma_{n}}(\xi) \neq 0$. So, by the commutative diagram (1), $\operatorname{Res}_{\mathbb{E}_{n}}^{\Gamma_{n}}(\xi) \neq 0$ iff $\operatorname{Res}_{U_{n} \times \mathbb{E}_{n}}^{\mathcal{G}_{n}}(X) \neq 0$, which is equivalent to the fact that the $s_{i}$ 's are not all equal to zero, or equivalently, $x \xi \notin(y)$. The proposition follows.

For convenience, given a central extension of groups

$$
1 \rightarrow A \rightarrow K \rightarrow C \rightarrow 1,
$$

denote by $\left\{E_{r}(K), d_{r}\right\}$ the Hochschild-Serre spectral sequence corresponding to the extension $(K)$. We now recall some results given in [12, 13] (see also [2] for $n=1$ ) concerning $\left\{E_{r}\left(\Gamma_{n}\right), d_{r}\right\}$. As usual, denote by $\mathcal{P}^{i}$ the Steenrod operations. Set $Z=i\left(\mathbb{F}_{p}\right) \subset C_{p^{2}} \subset \Gamma_{n}$. So $v=\operatorname{Res}_{Z}^{C_{p^{2}}}(w)$ is a generator of $H^{2}(Z)$. Let

$$
\begin{aligned}
X_{n} & =x_{1} x_{2} \ldots x_{2 n-1} x_{2 n}, \\
\eta_{i} & =\mathcal{P}^{p^{i-2}} \ldots \mathcal{P}^{1} \beta z \\
& =\sum_{j=1}^{n}\left(x_{2 j-1} y_{2 j}^{p^{i-1}}-x_{2 j} y_{2 j-1}^{p^{i-1}}\right), \\
\xi_{m} & =\beta \mathcal{P}^{p^{m-1}} \ldots \mathcal{P}^{1} \beta z \\
& =\sum_{j=1}^{n}\left(y_{2 j-1} y_{2 j}^{p^{m}}-y_{2 j} y_{2 j-1}^{p^{m}}\right),
\end{aligned}
$$

$1 \leq i \leq n+1,1 \leq m \leq n$, be elements of $H^{*}(V)$. We have

Theorem 1 ([2], as corrected in [13, Rk. 2.11(ii)], [12]). (i) We have

$$
\begin{aligned}
E_{\infty}\left(\Gamma_{1}\right)= & H^{*}(V) /\left(z, \eta_{1}, \eta_{2}, \xi_{1}\right) \otimes \mathbb{F}_{p}\left[v^{p}\right] \\
& \oplus\left(\mathbb{F}_{p} X_{1} \oplus \mathbb{F}_{p} x X_{1}\right) \otimes \sum_{i=1}^{p-2} \mathbb{F}_{p}\left[v^{p}\right] v^{i} .
\end{aligned}
$$


(ii) For $n \geq 2$,

$$
\begin{gathered}
\begin{array}{c}
E_{2 p+1}\left(\Gamma_{n}\right)=H^{*}(V) /\left(z, \eta_{1}, \xi_{1}, A_{n} \eta_{2}\right) \otimes \mathbb{F}_{p}\left[v^{p}\right] \\
\oplus\left(\mathbb{F}_{p} X_{n} \oplus \mathbb{F}_{p} x X_{n}\right) \otimes \sum_{i=1}^{p-2} \mathbb{F}_{p}\left[v^{p}\right] v^{i}
\end{array} \\
\text { with } A_{n}=\sum_{i=1}^{n} x_{1} x_{2} \ldots \hat{x}_{2 i-1} \hat{x}_{2 i} \ldots x_{2 n-1} x_{2 n} .
\end{gathered}
$$

Let $W$ be the vector subspace of $V$ given by $W=\operatorname{Ker}\left(x-x_{1}\right)$. We then have the central extension

$$
1 \rightarrow Z \rightarrow \mathbb{M}_{n} \rightarrow W \rightarrow 0
$$

with factor set $z^{\prime}=z_{n}^{\prime}=y_{1}+x_{1} x_{2}+\cdots+x_{2 n-1} x_{2 n}$. Following [10], [12], we also have

Proposition 2 ([10, 12]). (i) We have

$$
\begin{aligned}
E_{\infty}\left(\mathbb{M}_{1}\right)=E_{2 p+1}\left(\mathbb{M}_{1}\right)= & H^{*}(W) /\left(z^{\prime}, \beta z^{\prime}\right) \otimes \mathbb{F}_{p}\left[v^{p}\right] \\
& \oplus\left(\mathbb{F}_{p}\left[y_{2}\right] x_{1} \oplus \mathbb{F}_{p}\left[y_{2}\right] x_{1} x_{2}\right) \otimes \mathbb{F}_{p}\left[v^{p}\right] v^{p-1} \\
& \oplus\left(\mathbb{F}_{p} x_{1} \oplus \mathbb{F}_{p} x_{1} x_{2}\right) \otimes \sum_{i=1}^{p-2} \mathbb{F}_{p}\left[v^{p}\right] v^{i}
\end{aligned}
$$

(ii) For $n \geq 2$,

$$
\begin{aligned}
E_{2 p+1}\left(\mathbb{M}_{n}\right)= & H^{*}(W) /\left(z^{\prime}, \beta z^{\prime}, \beta \mathcal{P}^{1} \beta z^{\prime}, B_{n} \cdot \mathcal{P}^{1} \beta z^{\prime}\right) \otimes \mathbb{F}_{p}\left[v^{p}\right] \\
& \oplus\left(\mathbb{F}_{p} x_{1} x_{3} x_{4} \ldots x_{2 n-1} x_{2 n} \oplus \mathbb{F}_{p} X_{n}\right) \otimes \sum_{i=1}^{p-2} \mathbb{F}_{p}\left[v^{p}\right] v^{i}
\end{aligned}
$$

with $B_{n}=\sum_{i=2}^{n} x_{1} x_{3} x_{4} \ldots \hat{x}_{2 i-1} \hat{x}_{2 i} \ldots x_{2 n-1} x_{2 n}$.

We also prove

Proposition 3. If $\xi \in H^{*}\left(\Gamma_{n}\right)$ and $|\xi|<2 n+2$, then $\xi \in \operatorname{Im}_{\operatorname{Inf}_{\Gamma_{n}}^{V}}^{V}$.

The proof of the proposition is divided into the following lemmas. Set

$$
\mathcal{R}=E\left[x_{1}, \ldots, x_{m}\right] \otimes \mathbb{F}_{p}\left[t_{1}, \ldots, t_{m}\right],
$$

and let

$$
\alpha_{i}=\sum_{j=1}^{m} x_{j} t_{j}^{p^{i-1}}, \quad 1 \leq i \leq m,
$$

be elements of $\mathcal{R}$. Denote by $\mathcal{I}_{k, m}$ the set consisting of subsets of $k$ elements of $\{1, \ldots, m\}$. For every element $I=\left\{i_{1}, \ldots, i_{k}\right\}$ of $\mathcal{I}_{k, m}$ with $i_{1}<\cdots<i_{k}$, set $x_{I}=x_{i_{1}} \ldots x_{i_{k}}$ and $x_{\emptyset}=1$.

Lemma 2. For $X \in \mathcal{R}$ and $1 \leq k \leq m$,

(i) if $X \cdot \alpha_{1} \ldots \alpha_{k}=0$, then $X \in\left(\alpha_{1}, \ldots, \alpha_{k}, x_{I} \mid I \in \mathcal{I}_{m-k+1, m}\right)$;

(ii) if $X \cdot \alpha_{k}=0$, then $X \in\left(\alpha_{k}, x_{1} \ldots x_{m}\right)$. 
Proof. (i) We argue by induction on $m$. The case $m=2$ is obvious. Assume that (i) holds for $m-1$.

If $k=m$, then

$$
\alpha_{1} \ldots \alpha_{m}=\left|\begin{array}{ccc}
t_{1} & \ldots & t_{m} \\
t_{1}^{p} & \ldots & t_{m}^{p} \\
\cdot & \ldots & \cdot \\
t_{1}^{p^{m-1}} & \ldots & t_{m}^{p^{m-1}}
\end{array}\right| x_{1} \ldots x_{m}
$$

so $X \in\left(x_{i} \mid 1 \leq i \leq m\right)$. Suppose that $k<m$. Write

$$
\alpha_{i}=\alpha_{i}^{\prime}+x_{m} t_{m}^{i-1}
$$

with $\alpha_{i}^{\prime}=\sum_{j=1}^{m-1} x_{j} t_{j}^{p^{i-1}}, 1 \leq i \leq m$, and

$$
X=X^{\prime}+X^{\prime \prime} x_{m}
$$

with $X^{\prime}, X^{\prime \prime}$ free of $x_{m}$. Since $X \alpha_{1} \ldots \alpha_{k}=0$, we have

$$
\begin{aligned}
& 0=X^{\prime} \alpha_{1}^{\prime} \ldots \alpha_{k}^{\prime}, \\
& 0=(-1)^{k} X^{\prime \prime} \alpha_{1}^{\prime} \ldots \alpha_{k}^{\prime}+X^{\prime} \sum_{i=1}^{k}(-1)^{k-i} t_{m}^{p^{i-1}} \alpha_{1}^{\prime} \ldots \widehat{\alpha}_{i}^{\prime} \ldots \alpha_{k}^{\prime} .
\end{aligned}
$$

By writing

$$
\begin{aligned}
X^{\prime} & =t_{m}^{r_{1}} f_{1}+\cdots+t_{m}^{r_{j}} f_{j}, \\
X^{\prime \prime} & =t_{m}^{s_{1}} g_{1}+\cdots+t_{m}^{s_{i}} g_{i}
\end{aligned}
$$

with $f_{i}, g_{j}$ free of $t_{m}, r_{1}<\cdots<r_{j}, s_{1}<\cdots<s_{i}$, we have

$$
(-1)^{k} t_{m}^{s_{i}} g_{i} \alpha_{1}^{\prime} \ldots \alpha_{k}^{\prime}+t_{m}^{p^{k-1}+r_{j}} f_{j} \alpha_{1}^{\prime} \ldots \alpha_{k-1}^{\prime}=0 .
$$

Consider the following cases:

- $r_{j}+p^{k-1}>s_{i}$ : from (2), $f_{j} \alpha_{1}^{\prime} \ldots \alpha_{k-1}^{\prime}=0$. By the inductive hypothesis, $f_{j} \in\left(\alpha_{1}^{\prime}, \ldots, \alpha_{k-1}^{\prime}, \mathcal{I}_{m-k+1, m-1}\right)$. Since $\alpha_{i}^{\prime}=\alpha_{i}-x_{m} t_{m}^{p^{i-1}}$, we have $X=t_{m}^{r_{1}} f_{1}+$ $\cdots+t_{m}^{r_{j-1}} f_{j-1} \bmod \left(x_{m}, \alpha_{1}, \ldots, \alpha_{k}, \mathcal{I}_{m-k+1, m}\right)$. So we may suppose that $f_{j}=0$.

- $r_{j}+p^{k-1}<s_{i}$ : from $(2), g_{i} \alpha_{1}^{\prime} \ldots \alpha_{k}^{\prime}=0$. By the inductive hypothesis, $g_{i} \in\left(\alpha_{1}^{\prime}, \ldots, \alpha_{k}^{\prime}, \mathcal{I}_{m-k, m-1}\right)$. So $x_{m} g_{i} \in\left(\alpha_{1}, \ldots, \alpha_{k}, \mathcal{I}_{m-k+1, m}\right)$.

- $r_{j}+p^{k-1}=s_{i}$ : from $(2),\left((-1)^{k+k-1} g_{i} \alpha_{k}^{\prime}+f_{j}\right) \alpha_{1}^{\prime} \ldots \alpha_{k-1}^{\prime}=0$. By the inductive hypothesis, $f_{j}=g_{i} \alpha_{k}^{\prime} \bmod \left(\alpha_{1}^{\prime}, \ldots, \alpha_{k-1}^{\prime}, \mathcal{I}_{m-k+1, m-1}\right)$. Since $\alpha_{i}^{\prime}=\alpha_{i}-x_{m} t_{m}^{p^{i-1}}$, we have $f_{j}=g_{i} \alpha_{k}^{\prime} \bmod \left(x_{m}, \alpha_{1}, \ldots, \alpha_{k-1}, \mathcal{I}_{m-k+1, m}\right)$. So we may suppose that $f_{j}=g_{i} \alpha_{k}^{\prime}$. Since

$$
\begin{aligned}
t_{m}^{r_{j}} f_{j}+t_{m}^{s_{i}} g_{i} x_{m} & =t_{m}^{r_{j}}\left(f_{j}+t_{m}^{p^{k-1}} g_{i} x_{m}\right) \\
& =t_{m}^{r_{j}} g_{i}\left(\alpha_{k}^{\prime}+t_{m}^{p^{k-1}} x_{m}\right) \\
& =t_{m}^{r_{j}} g_{i} \alpha_{k},
\end{aligned}
$$

we may then suppose that $f_{j}=0$ and $g_{i}=0$.

The above arguments show that we may reduce to the case $X^{\prime}=0$. It follows that $X^{\prime \prime} \alpha_{1}^{\prime} \ldots \alpha_{k}^{\prime}=0$. By the inductive hypothesis, $X^{\prime \prime} \in\left(\alpha_{1}^{\prime}, \ldots, \alpha_{k}^{\prime}, \mathcal{I}_{m-k, m-1}\right)$. Hence $x_{m} X^{\prime \prime} \in\left(\alpha_{1}, \ldots, \alpha_{k}, \mathcal{I}_{m-k+1, m}\right)$. (i) is proved. 
(ii) We again use induction on $m$. The case $m=1$ is trivial. Assume that (ii) holds for $m-1$. As above, write

$$
X=X^{\prime}+X^{\prime \prime} x_{m}
$$

with $X^{\prime}, X^{\prime \prime}$ free of $x_{m}$. Arguing as above, we may reduce to the case $X^{\prime}=0$. It follows that $X^{\prime \prime} \alpha_{k}^{\prime}=0$. Bu the inductive hypothesis, $X^{\prime \prime} \in\left(\alpha_{k}^{\prime}, x_{1} \ldots x_{m-1}\right)$. Hence $x_{m} X^{\prime \prime} \in\left(\alpha_{k}, x_{1} \ldots x_{m}\right)$.

The lemma is proved.

Lemma 3. Let $1 \leq k \leq n$ and let $Y_{1}, \ldots, Y_{k}$ be elements of $H^{*}(V)$.

(i) If $Y_{1} \xi_{1}+\cdots+Y_{k} \xi_{k}=0$, then $Y_{k} \in\left(\xi_{1}, \ldots, \xi_{k-1}\right)$.

(ii) Assume that

$$
Y_{k}=\sum_{\substack{I \subset\{1, \ldots, 2 n\} \\ \#(I)<2 n-k+1}} x_{I} f_{I}\left(y, y_{1}, \ldots, y_{2 n}\right)
$$

We have:

(iia) if $Y_{1} \eta_{1}+\cdots+Y_{k} \eta_{k}=0$, then $Y_{k} \in\left(\eta_{1}, \ldots, \eta_{k}\right)$;

(iib) if $Y_{k} \in \bigcap_{i=1}^{k}\left(\eta_{i}\right)$, then $Y_{k} \in\left(\eta_{1} \ldots \eta_{k}\right)$;

(iic) if $Y_{1} \xi_{1}+\cdots+Y_{k-1} \xi_{k-1}+Y_{k} \eta_{\ell}=0$ with $1 \leq \ell \leq n$, then

$$
Y_{k} \in\left(\eta_{\ell}, \xi_{1}, \ldots, \xi_{k-1}\right) \text {. }
$$

Proof. (i) For $1 \leq i \leq k$, write

$$
Y_{i}=\sum_{I \subset\{1, \ldots, 2 n\}} x_{I} f_{I}^{(i)}\left(y, y_{1}, \ldots, y_{2 n}\right) .
$$

Then, for every $I$, we have

$$
\sum_{i=1}^{k} f_{I}^{(i)} \xi_{i}=0
$$

According to [18], $\xi_{1}, \ldots, \xi_{k}$ is a regular sequence in $P$. So the above equality implies $f_{I}^{(k)} \in\left(\xi_{1}, \ldots, \xi_{k-1}\right)$. Therefore $Y_{k} \in\left(\xi_{1}, \ldots, \xi_{k-1}\right)$.

(iia) It follows that $Y_{k} \eta_{1} \ldots \eta_{k}=0$. By Lemma $2, Y_{k} \in\left(\eta_{1}, \ldots, \eta_{k}, \mathcal{I}_{2 n-k+1,2 n}\right)$. So $Y_{k} \in\left(\eta_{1}, \ldots, \eta_{k}\right)$.

(iib) We use induction on $k$. For $k=2, X \eta_{1}+Y \eta_{2}=0$ implies $Y \eta_{1} \eta_{2}=0$. By Lemma 2, $Y \in\left(\eta_{1}, \eta_{2}, \mathcal{I}_{2 n-1,2 n}\right)$. So $Y \in\left(\eta_{1}, \eta_{2}\right)$. Write $Y=a \eta_{1}+b \eta_{2}$. Then $Y_{2}=Y \eta_{2}=a \eta_{1} \eta_{2}$.

Assume that (iib) holds for $k-1 \geq 2$. As $Y_{k} \in \bigcap_{i<k}\left(\eta_{i}\right)$, it follows from the inductive hypothesis that $Y_{k}=Y \eta_{1} \ldots \eta_{k-1}$. Write $Y_{k}=X \eta_{k}$. Then $Y \eta_{1} \ldots \eta_{k}=0$. By Lemma 2, $Y=c_{1} \eta_{1}+\cdots+c_{k} \eta_{k}$. So $Y_{k}=(-1)^{k-1} c_{k} \eta_{1} \ldots \eta_{k}$.

(iic) Again, we use induction on $k$. $Y_{1} \xi_{1}+Y_{2} \eta_{\ell}=0$ implies $Y_{1} \eta_{\ell}=0$. By Lemma $2, Y_{1} \in\left(\eta_{\ell}\right)$. Write $Y_{1}=c \eta_{\ell}$. Then $\left(c \xi_{1}+Y_{2}\right) \eta_{\ell}=0$. By Lemma $2, c \xi_{1}+Y_{2} \in\left(\eta_{\ell}\right)$; hence $Y_{2} \in\left(\eta_{\ell}, \xi_{1}\right)$.

Assume that (iic) holds for $k-1 \geq 2$. As $Y_{1} \eta_{\ell} \xi_{1}+\cdots+Y_{k-1} \eta_{\ell} \xi_{k-1}=0$, it follows from (i) that $Y_{k-1} \eta_{\ell} \in\left(\xi_{1}, \ldots, \xi_{k-2}\right)$. By the inductive hypotheses, we may write $Y_{k-1}=c_{1} \xi_{1}+\cdots+c_{k-2} \xi_{k-2}+c_{k-1} \eta_{\ell}$. Hence

$$
\left(Y_{1}+c_{1} \xi_{k-1}\right) \xi_{1}+\cdots+\left(Y_{k-2}+c_{k-2} \xi_{k-1}\right) \xi_{k-2}+\left(Y_{k}+c_{k-1} \xi_{k-1}\right) \eta_{\ell}=0 .
$$

By the inductive hypothesis, $Y_{k}+c_{k-1} \xi_{k-1} \in\left(\xi_{1}, \ldots, \xi_{k-2}, \eta_{\ell}\right)$, and hence $Y_{k} \in$ $\left(\xi_{1}, \ldots, \xi_{k-1}, \eta_{\ell}\right)$. 
For $1 \leq i \leq n+1,0 \leq k \leq n$, denote by $\Delta_{i, k}$ the ideal of $H^{*}(V)$ given by

$$
\Delta_{i, k}= \begin{cases}\left(z, \eta_{j}, \xi_{m} \mid 1 \leq j \leq i, 1 \leq m \leq k\right) & \text { if } k \geq 1, \\ \left(z, \eta_{j} \mid 1 \leq j \leq i\right) & \text { if } k=0 .\end{cases}
$$

Lemma 4. If $X=\sum_{\#(I)<2 n-2 k+1} x_{I} X_{I}\left(y_{1}, \ldots, y_{2 n}\right)$ and $X \xi_{j} \in \Delta_{k, j-1}$ with $1 \leq$ $j \leq k \leq n$, then $X \in \Delta_{k, j-1}$.

Proof. Write

$$
X \xi_{j}=a_{0} z+\sum_{i=1}^{k} a_{i} \eta_{i}+\sum_{i=1}^{j-1} b_{i} \xi_{i}
$$

with $a_{i}, b_{\ell} \in H^{*}(V)$. Since $y=z-x_{1} x_{2}-\cdots-x_{2 n-1} x_{2 n}$, we may suppose that $a_{i}, b_{\ell}, 1 \leq i \leq k, 1 \leq \ell \leq j-1$, are free of $y$. It follows that $a_{0}=0$ and

$$
X \xi_{j} \eta_{1} \ldots \eta_{k}=\sum_{i=1}^{j-1} b_{i} \xi_{i} \eta_{1} \ldots \eta_{k}
$$

We now argue by induction on $j$. For $j=1$, it follows that $X \xi_{1} \eta_{1} \ldots \eta_{k}=0$. Hence $X \eta_{1} \ldots \eta_{k}=0$. By Lemma $2, X \in\left(\eta_{1}, \ldots, \eta_{k}\right)$.

Assume that the lemma holds for $j-1 \geq 1$. By Lemma 3 (i) and by (3), there exists $c_{i} \in H^{*}(V)$ such that

$$
X \eta_{1} \ldots \eta_{k}=c_{1} \xi_{1}+\cdots+c_{j-1} \xi_{j-1} .
$$

Therefore, by Lemma 3 (i), $c_{j-1} \eta_{i} \in\left(\xi_{1}, \ldots, \xi_{j-2}\right)$, for every $1 \leq i \leq k$; by Lemma 3 (iic), $c_{j-1} \in \bigcap_{i \leq k}\left(\xi_{1}, \ldots, \xi_{j-2}, \eta_{i}\right)$. By writing

$$
\begin{aligned}
c_{j-1} & =d_{1} \xi_{1}+\cdots+d_{j-2} \xi_{j-2}+d \eta_{1} \ldots \eta_{i-1} \\
& =e_{1} \xi_{1}+\cdots+e_{j-2} \xi_{j-2}+e \eta_{i},
\end{aligned}
$$

we get

$$
\left[\left(e_{1}-d_{1}\right) \xi_{1}+\cdots+\left(e_{j-2}-d_{j-2}\right) \xi_{j-2}\right] \eta_{1} \ldots \eta_{i}=0 .
$$

By Lemma $2,\left(e_{1}-d_{1}\right) \xi_{1}+\cdots+\left(e_{j-2}-d_{j-2}\right) \xi_{j-2}$ contains $\eta_{1} \ldots \eta_{i}$ as a factor. Hence $e \eta_{i} \in \bigcap_{\ell \leq i}\left(\eta_{\ell}\right)$. By Lemma 3 (iib), $c_{j-1} \in\left(\xi_{1}, \ldots, \xi_{j-2}, \eta_{1} \ldots \eta_{i}\right)$. So we may suppose that $c_{j-1} \in\left(\eta_{1} \ldots \eta_{k}\right)$. By writing $c_{j-1}=c \eta_{1} \ldots \eta_{k}$, we have

$$
\left(X-c \xi_{j-1}\right) \eta_{1} \ldots \eta_{k}=c_{1} \xi_{1}+\cdots+c_{j-2} \xi_{j-2}
$$

By the inductive hypothesis, this implies $X-c \xi_{j-1} \in \Delta_{k, j-2}$. So $X \in \Delta_{k, j-1}$. The lemma follows.

Lemma 5. If $X=\sum_{\#(I)<2 n-2 k} x_{I} X_{I}\left(y_{1}, \ldots, y_{2 n}\right)$ and $X \eta_{k} \in \Delta_{k-1, k-1}$ with $1 \leq$ $k \leq n+1$, then $X \in \Delta_{k, k-1}$.

Proof. Write

$$
X \eta_{k}=a_{0} z+\sum_{i=1}^{k-1}\left(a_{i} \eta_{i}+b_{i} \xi_{i}\right)
$$

Arguing as in the proof of Lemma 4 , we may suppose that $a_{i}, b_{i}$, with $1 \leq i \leq k-1$, are free of $y$. It follows that $a_{0}=0$ and

$$
X \eta_{k}=\sum_{i=1}^{k-1}\left(a_{i} \eta_{i}+b_{i} \xi_{i}\right) .
$$


Furthermore, we may suppose that every $b_{i}$ is of form

$$
b_{i}=\sum_{\#(I)<2 n-2 k+1} x_{I} b_{I}^{(i)} .
$$

Therefore, applying Lemma 4 yields $b_{k-1} \in \Delta_{k, k-2}$. Hence, by induction, we need only consider the case

$$
X \eta_{k}=b_{1} \xi_{1}+\sum_{i=1}^{k-1} a_{i} \eta_{i}
$$

This implies $b_{1} \xi_{1} \eta_{1} \ldots \eta_{k}=0$. So $b_{1} \eta_{1} \ldots \eta_{k}=0$. By Lemma $2, b_{1} \in\left(\eta_{1}, \ldots, \eta_{k}\right)$. The lemma follows.

Let us now consider the Hochschild-Serre spectral sequence $\left\{E_{r}\left(\Gamma_{n}\right), d_{r}\right\}$. It follows that, for $k<2 n+2$,

$$
\sum_{i+j=k} E_{2 p+1}^{i, j} \subset E_{2 p+1}^{k, 0} \oplus \bigoplus_{r \geq 1} E_{2 p+1}^{*, 2 p r} .
$$

By Kudo's transgression theorem, for $m \leq n, 1 \otimes v^{p^{m}}$ (resp. $\left.\eta_{m} \otimes v^{p^{m-1}(p-1)}\right)$ survives to $E_{2 p^{m}+1}\left(\right.$ resp. $\left.E_{2 p^{m-1}(p-1)+1}\right)$ and

$$
\begin{aligned}
d_{2 p^{m}+1}\left(1 \otimes v^{p^{m}}\right) & =\eta_{m+1}, \\
d_{2 p^{m-1}(p-1)+1}\left(\eta_{m} \otimes v^{p^{m-1}(p-1)}\right) & =-\xi_{m} .
\end{aligned}
$$

Lemma 6. For $k<2 n+2$ and $1 \leq m \leq n$, we have

$$
\sum_{i+j=k} E_{2 p^{m}+1}^{i, j} \subset E_{2 p^{m}+1}^{k, 0} \oplus \bigoplus_{r \geq 1} E_{2 p^{m}+1}^{*, 2 p^{m} r}
$$

Proof. By the structure of $E_{2 p+1}\left(\Gamma_{n}\right)$, the lemma holds for $m=1$. Suppose that the lemma holds for $m=s \geq 1$. Let $\psi=X \otimes v^{\ell p^{s}}$ be an element of $E_{2}\left(\Gamma_{n}\right)$ surviving to $E_{2 p^{s+1}+1}$, with $1 \leq \ell \leq p-1$ and $|X|+2 \ell p^{s}=k<2 n+2$. So $d_{2 p^{s}+1}(\psi)=\ell X \eta_{s+1} \otimes v^{(\ell-1) p^{s}}$ must be hit by images under the differentials of elements of degrees less than $2 n+2$. By the inductive hypothesis and by Kudo's theorem, these images belong to the ideal $\Delta_{s, s}$; hence so does $X \eta_{s+1}$. Since in $E_{3}\left(\Gamma_{n}\right)$ we have $y=-\left(x_{1} x_{2}+\cdots+x_{2 n-1} x_{2 n}\right)$, we may suppose that $X$ is free of $y$. As $|X|<2 n+2-2 p^{s}<2 n-2 s-2$, by Lemma 5 , this means that $X \in \Delta_{s+1, s}$. So $\psi=0$ in $E_{2 p^{s}+2}$ if $\ell<p-1$. If $\ell=p-1$, write $\psi=Y \eta_{s+1} \otimes v^{p^{s}(p-1)}$. Then $d_{2 p^{s}(p-1)+1}(\psi)=-Y \xi_{s+1} \in \Delta_{s+1, s}$. Arguing as above, we may suppose that $Y$ is free of $y$. By Lemma 4 , as $|\psi|<2 n+2$, we have $Y \in \Delta_{s+1, s}$. So $\psi=0$ in $E_{2 p^{s}(p-1)+2}$. The lemma follows.

Proof of Proposition 3. It follows from Lemma 6 that $\xi$ either belongs to $\operatorname{Im}_{\operatorname{Inf}} \Gamma_{\Gamma_{n}}$ or represents an element of $E_{\infty}^{*, 2 p^{n} r}$. As $2 p^{n}>2 n+2$, the fact that $|\xi|<2 n+2$ implies $\xi \in \operatorname{Im}_{\operatorname{Inf}}^{V} \Gamma_{n}$. The proposition follows.

\section{The CASE $\exp (G)>p$ OR $|G|=p^{5}$}

We first consider the case $G=\mathbb{M}_{n}$. Consider $G$ as a subgroup of $\Gamma_{n}$ by setting $G=\operatorname{Ker}\left(x-x_{1}\right)$. If $n=1$, it follows from [10] (see also [4]) that $H^{*}(\mathbb{M})$ contains 
a non-zero essential element, namely $X_{1}$. Assume inductively that $0 \neq X_{n-1} \in$ $\operatorname{Ess}\left(\mathbb{M}_{n-1}\right)$. As $\mathbb{M}_{n}=\mathbb{M}_{n-1} \bullet \mathbb{M}$, we have the following central extension:

$$
0 \rightarrow \mathbb{F}_{p} \rightarrow \mathbb{M}_{n-1} \times \mathbb{M} \rightarrow \mathbb{M}_{n} \rightarrow 1
$$

The fact that $H^{*}\left(\mathbb{M}_{n}\right)$ contains non-zero essential elements follows from

Proposition 4. $0 \neq X_{n} \in \operatorname{Ess}\left(\mathbb{M}_{n}\right)$.

Proof. Let $K$ be a maximal subgroup of $\mathbb{M}_{n}$. As $\operatorname{dim}_{\mathbb{F}_{p}} H^{1}(K)=2 n-1$, it follows that the product of any $2 n$ elements of $H^{1}(K)$ vanishes. Hence $\operatorname{Res}_{K}^{\mathbb{M}_{n}}\left(X_{n}\right)=0$, which implies that $X_{n} \in \operatorname{Ess}\left(\mathbb{M}_{n}\right)$. Furthermore, as $\operatorname{Inf}_{\mathbb{M}_{n-1} \times \mathbb{M}}^{\mathbb{M}_{n}}\left(X_{n}\right)=X_{n-1} \times$ $x_{2 n-1} x_{2 n} \neq 0$ in $H^{*}\left(\mathbb{M}_{n-1} \times \mathbb{M}\right)$ by the inductive hypothesis, it follows that $X_{n} \neq 0$. The proposition is proved

By Theorem 1 (i), $X_{1}$ and $x X_{1}$ are non-zero elements of $H^{*}\left(\Gamma_{1}\right)$. By considering the central extension $0 \rightarrow \mathbb{F}_{p} \rightarrow \Gamma_{n-1} \times \mathbb{M} \rightarrow \Gamma_{n} \rightarrow 1$, and by using the same argument given in the proof of Proposition 4, we also have

Proposition 5. The elements $x X_{n}$ and $X_{n}$ are non-zero elements of $H^{*}\left(\Gamma_{n}\right)$.

Our next task is to prove that the theorem holds for the extraspecial $p$-group $G=\mathbb{E}_{2}$. Consider $\mathbb{E}_{2}$ as a subgroup of $\Gamma_{2}$ as in Lemma 1. Let $Q$ be the element of $H^{*}(V)$ defined by $Q=Q_{2,1}^{1,2}-Q_{2,1}^{3,4}$ with

$$
Q_{2,1}^{i, j}=Q_{2,1}\left(y_{i}, y_{j}\right)=\frac{y_{i}^{p^{2}} y_{j}-y_{j}^{p^{2}} y_{i}}{y_{i}^{p} y_{j}-y_{j}^{p} y_{i}}
$$

(so $Q_{2,1}^{i, j}$ is nothing but the Dickson invariant of order $2\left(p^{2}-p\right)$ with variables $\left.y_{i}, y_{j}\right)$, and set $\eta=x_{1} x_{2} Q$. It follows from [19, Th. 8.25] that $0 \neq \eta \in H^{*}\left(\mathbb{E}_{2}\right)$. The case $G=\mathbb{E}_{2}$ is then proved by the following:

Proposition 6. $\eta \in \operatorname{Ess}\left(\mathbb{E}_{2}\right)$.

Proof. Let $K$ be a maximal subgroup of $\mathbb{E}_{2}$, so $K \cong \mathbb{E} \times C_{p}$. If $\operatorname{Res}_{K}^{\mathbb{E}_{2}}\left(x_{3} x_{4}\right)=0$, it is clear that $\operatorname{Res}_{K}^{\mathbb{E}_{2}}\left(x_{1} x_{2} Q_{2,1}^{1,2}\right)=0$; we can then assume that $\operatorname{Res}_{K}^{\mathbb{E}_{2}}\left(x_{3} x_{4}\right) \neq 0$. Choose a basis $u_{1}, u_{2}, u_{3}, u_{4}$ of $H^{1}\left(\mathbb{E}_{2} / Z\right)$ such that $K=\operatorname{Ker} u_{4}, x_{1} x_{2}+x_{3} x_{4}=u_{1} u_{2}+u_{3} u_{4}$, $\operatorname{Res}_{K}^{\mathbb{E}_{2}}\left(x_{1} x_{2}\right)=u_{1} u_{2}+u_{1} u_{3}$ and $\operatorname{Res}_{K}^{\mathbb{E}_{2}}\left(x_{3} x_{4}\right)=-u_{1} u_{3}$. This implies that $u_{1} u_{2}=0$ in $H^{*}(K)$. By setting $v_{i}=\beta u_{i}$, we have

$$
\begin{aligned}
\operatorname{Res}_{K}^{\mathbb{E}_{2}}(\eta) & =\left(u_{1} u_{2}+u_{1} u_{3}\right)\left(Q_{2,1}\left(v_{1}, v_{2}+v_{3}\right)-Q_{2,1}\left(v_{3}, v_{1}\right)\right) \\
& =u_{1} u_{3}\left(Q_{2,1}\left(v_{1}, v_{2}+v_{3}\right)-Q_{2,1}\left(v_{3}, v_{1}\right)\right) \quad \text { as } u_{1} u_{2}=0 \text { in } H^{*}(K) .
\end{aligned}
$$

Set $Y=Q_{2,1}\left(v_{1}, v_{2}+v_{3}\right)-Q_{2,1}\left(v_{3}, v_{1}\right)$. Following [13. Proof of Lemma 1.10], $Y$ contains $v_{2}^{p}-v_{2} v_{1}^{p-1}$ as a factor. As $u_{1}\left(v_{2}^{p}-v_{2} v_{1}^{p-1}\right)=-\mathcal{P}^{1} \beta\left(u_{1} u_{2}\right)+v_{1}^{p-1} \beta\left(u_{1} u_{2}\right)$, we have $\operatorname{Res}_{K}^{\mathbb{E}_{2}}(\eta)=0$. So $\eta \in E s s\left(\mathbb{E}_{2}\right)$. The proposition is proved.

For $\exp (G)>p$ or $|G|=p^{5}$, Propositions 4 and 6 tell us that there exist nonzero essential cohomology classes of $G$ which belong to $\operatorname{Im} \operatorname{Inf}_{G}^{V}$. Furthermore, if $G=\mathbb{M}_{2}$, then [12, Proposition 1.9] and [13, Theorem 3.10] tell us that

$$
x_{3} x_{4} N \text { and }\left(y_{3} x_{4}-y_{4} x_{3}\right) N
$$

are also non-zero elements of $\operatorname{Ess}\left(\mathbb{M}_{2}\right)$ with $N=\left(y_{2}^{p-1}-y_{3}^{p-1}\right)\left(y_{2}^{p-1}-y_{4}^{p-1}\right)$. We can then end the section by the following

Question. For $G \neq \mathbb{E}$, is it true that $\operatorname{Ess}(G) \cap \operatorname{Im}_{\operatorname{Inf}_{G}}^{V} \neq\{0\}$ ? 


\section{The CASE $\exp (G)=p$}

We first point out some mod- $p$ cohomology classes of $\Gamma_{n}$, by using the following argument given by D.J. Green [6]. Let $K$ be a $p$-group containing $C$ as a central subgroup. We have the central extension

$$
1 \rightarrow C \rightarrow K \stackrel{\mathrm{pr}}{\rightarrow} K / C \rightarrow 1
$$

On the other hand, by considering the extension

$$
(K \times C) \quad 1 \rightarrow C \stackrel{\ell}{\rightarrow} K \times C \stackrel{j}{\rightarrow} K \rightarrow 1
$$

with $\ell(c)=(1, c), j(k, c)=k, c \in C, k \in K$, we have the commutative diagram

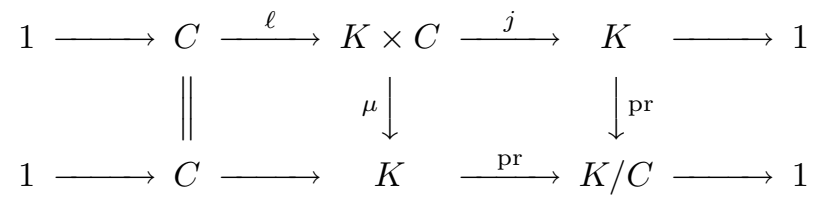

with $\mu(k, c)=k c, k \in K, c \in C$. The Hochschild-Serre spectral sequences corresponding to these extensions are of the forms

$$
\begin{aligned}
E_{2}(K) & =H^{*}(K / C) \otimes H^{*}(C) \Rightarrow H^{*}(K), \\
E_{2}(K \times C) & =E_{\infty}(K \times C)=H^{*}(K) \otimes H^{*}(C) .
\end{aligned}
$$

Furthermore, vertical arrows in (4) also induce a map $\left\{\mu_{r}: E_{r}(K) \rightarrow E_{r}(K \times C)\right\}$ between spectral sequences with $\mu_{2}=\left(\operatorname{Inf}_{K}^{K / C}, 1_{H^{*}(C)}\right)$.

The following is due to D.J. Green.

Proposition 7. For $r \geq 2$,

$$
\operatorname{Im}\left(d_{r}: E_{r}(K) \rightarrow E_{r}(K)\right) \subset \operatorname{Ker}_{\operatorname{Inf}_{K}^{K / C}} \otimes H^{*}(C) .
$$

Proof. Let $\xi \in E_{r}(K)$ and write $d_{r}(\xi)=\sum \phi_{j} \otimes \psi_{j}, \phi_{j} \in H^{*}(K / C), \psi_{j} \in H^{*}(C)$. We can suppose that the $\psi_{j}$ 's are linearly independent in $H^{*}(C)$. From the commutative diagram (4) and from the fact that $d_{r}: E_{r}(K \times C) \rightarrow E_{r}(K \times C)$ vanishes, we have

$$
\sum \operatorname{Inf}_{K}^{K / C}\left(\phi_{j}\right) \otimes \psi_{j}=\mu_{r}\left(d_{r}(\xi)\right)=d_{r}\left(\mu_{r}(\xi)\right)=0 .
$$

So $\phi_{j} \in \operatorname{Ker} \operatorname{Inf}_{K}^{K / C}$. The proposition follows.

Since $d_{2 p+1}\left(v^{p}\right)=\eta_{2}$ in $E_{2 p+1}\left(\Gamma_{n}\right)$ (resp. $\mathcal{P}^{1} \beta z^{\prime}$ in $E_{2 p+1}\left(\mathbb{M}_{n}\right)$ ), it follows from Theorem 1 and Proposition 2 that $A_{n} \otimes v^{p} \in E_{2 p+2}\left(\Gamma_{n}\right)$ and $B_{n} \otimes v^{p} \in E_{2 p+2}\left(\mathbb{M}_{n}\right)$. We then get

Proposition 8. For $1 \leq i \leq p-2$,

(i) if $n \geq 2$ then $x_{1} x_{3} x_{4} \ldots x_{2 n-1} x_{2 n} \otimes v^{i}, X_{n} \otimes v^{i}, x_{3} x_{4} \ldots x_{2 n-1} x_{2 n} \otimes v^{p}$ and $B_{n} \otimes v^{p}$ represent non-zero elements of $E_{\infty}\left(\mathbb{M}_{n}\right)$;

(ii) $X_{n} \otimes v^{i}, x X_{n} \otimes v^{i}$ and $A_{n} \otimes v^{p}$ represent non-zero elements of $E_{\infty}\left(\Gamma_{n}\right)$.

Proof. Note that, in $H^{*}(W)$, we have

$$
\begin{aligned}
x_{3} x_{4} \ldots x_{2 n-1} x_{2 n} \cdot \mathcal{P}^{1} \beta z^{\prime} & =\left(x_{3} x_{4} \ldots x_{2 n-1} x_{2 n}\right)\left(y_{1}^{p} x_{2}-y_{2}^{p} x_{1}\right) \\
& =\left(x_{3} x_{4} \ldots x_{2 n-1} x_{2 n}\right)\left(z^{\prime p} x_{2}+y_{2}^{p-1} \beta z^{\prime}-y_{2}^{p-1} y_{1} x_{2}\right) \\
& =\left(x_{3} x_{4} \ldots x_{2 n-1} x_{2 n}\right)\left(z^{\prime p} x_{2}+y_{2}^{p-1} \beta z^{\prime}-y_{2}^{p-1} z^{\prime} x_{2}\right) \\
& \in\left(z^{\prime}, \beta z^{\prime}\right) .
\end{aligned}
$$


So $d_{2 p+1}\left(x_{3} x_{4} \ldots x_{2 n-1} x_{2 n} \otimes v^{p}\right)=0$. Therefore $x_{3} x_{4} \ldots x_{2 n-1} x_{2 n} \otimes v^{p}$ survives to $E_{\infty}\left(\mathbb{M}_{n}\right)$.

By Proposition $4, X_{n} \neq 0$ in $H^{*}\left(\mathbb{M}_{n}\right)$ implies that $X_{n}, x_{1} x_{3} x_{4} \ldots x_{2 n-1} x_{2 n}$ and $B_{n}$ are not elements of Ker $\operatorname{Inf}_{\mathbb{M}_{n}}^{W}$. Similarly, Proposition 5 shows that $X_{n}, x X_{n}$ and $A_{n}$ are not elements of $\operatorname{Ker} \operatorname{Inf}_{\Gamma_{n}}^{V}$. The proposition follows from Proposition 7 .

For $n \geq 1$ and for $1 \leq i \leq p-2$, let us pick elements $X_{n, i} \in H^{2(n+i)-1}\left(\mathbb{M}_{n}\right)$ and $Y_{n, i} \in H^{2(n+i)}\left(\Gamma_{n}\right)$ which represent respectively $x_{1} x_{3} x_{4} \ldots x_{2 n-1} x_{2 n} \otimes v^{i} \in$ $E_{\infty}\left(\mathbb{M}_{n}\right)$ and $X_{n} \otimes v^{i} \in E_{\infty}\left(\Gamma_{n}\right)$; for $n \geq 2$, pick elements $X_{n, p-1} \in H^{2(n+p)-3}\left(\mathbb{M}_{n}\right)$, $Z_{n, p-1} \in H^{2(n+p)-2}\left(\mathbb{M}_{n}\right)$ and $Y_{n, p-1} \in H^{2(n+p)-2}\left(\Gamma_{n}\right)$ which represent respectively $B_{n} \otimes v^{p} \in E_{\infty}\left(\mathbb{M}_{n}\right), x_{3} x_{4} \ldots x_{2 n-1} x_{2 n} \otimes v^{p} \in E_{\infty}\left(\mathbb{M}_{n}\right)$ and $A_{n} \otimes v^{p} \in E_{\infty}\left(\Gamma_{n}\right)$ (the existence of such elements follows from Propositions 2 and 8). In particular, define $Y_{1, p-1}$ by

$$
Y_{1, p-1}=\mathcal{N}_{\text {Ker } x_{2} \rightarrow \Gamma_{1}}(w)
$$

with $\mathcal{N}$ the Evens norm map (note that Ker $x_{2} \cong C_{p^{2}} \times C_{p} \subset \Gamma_{1}$, so, by the Künneth formula, $w$ can be considered as an element of $H^{2}\left(\operatorname{Ker} x_{2}\right)$ ).

We now define the following subgroups of $\Gamma_{n}$ :

$$
\begin{aligned}
\mathbb{M}_{n} & =\operatorname{Ker}\left(x-x_{1}\right), \\
\Gamma_{n-1}^{\prime} & =\operatorname{Ker} x_{2 n} \cong \Gamma_{n-1} \times C_{p}, \\
\mathbb{M}_{n-1}^{\prime} & =\operatorname{Ker} x_{2 n} \cap \operatorname{Ker}\left(x-x_{1}\right) \quad\left(\text { so } \mathbb{M}_{n-1}^{\prime} \cong \mathbb{M}_{n-1} \times C_{p} \text { for } n>1\right), \\
' \Gamma_{n-1} & =\operatorname{Ker} x_{2} \cap \operatorname{Ker}\left(x-x_{1}\right) \cong \Gamma_{n-1}, \\
' \Gamma_{n-2} & =\operatorname{Ker} x_{2} \cap \operatorname{Ker} x_{2 n} \cap \operatorname{Ker}\left(x-x_{1}\right) \cong \Gamma_{n-2} \times C_{p}(\text { for } n \geq 2),
\end{aligned}
$$

with the convention that $\Gamma_{0}=C_{p^{2}}$. Therefore ${ }^{\prime} \Gamma_{n-2}=\mathbb{M}_{n-1}^{\prime} \cap{ }^{\prime} \Gamma_{n-1}$ and ' $\Gamma_{0}=$ $C_{p^{2}} \times C_{p}$. If $K$ is one of the above subgroups, then $K$ contains $Z$ as a central subgroup and we have the central extension

$$
1 \rightarrow Z \rightarrow K \rightarrow K / Z \rightarrow 1 .
$$

For convenience, we also define the elements $Y_{0, i} \in H^{2 i}\left(\Gamma_{0}\right), 1 \leq i \leq p-1$, by $Y_{0, i}=w^{i}$. With some abuse of notation, by the Künneth formula, the $Y_{n-1, i}$ (resp. $\left.X_{n-1, i}, Z_{n-1, i}\right)$ 's are considered as elements of $H^{*}\left(\Gamma_{n-1}^{\prime}\right), H^{*}\left({ }^{\prime} \Gamma_{n-1}\right)$ and $H^{*}\left({ }^{(} \Gamma_{n-1}\right)$ (resp. $\left.H^{*}\left(\mathbb{M}_{n-1}^{\prime}\right)\right)$. We have

Lemma 7. For $n \geq 1$ and $1 \leq i \leq p-1,1 \leq j \leq p-2$,

(i) $\operatorname{Res}_{\mathbb{M}_{n}^{n}}^{\Gamma_{n}}\left(Y_{n, j}\right)+x_{2} X_{n, j} \in \operatorname{Im}_{\operatorname{Inf}_{\mathbb{M}_{n}}}^{W}$; if $n>1$ then $\operatorname{Res}_{\mathbb{M}_{n}^{n}}^{\Gamma_{n}}\left(Y_{n, p-1}\right)+x_{2} X_{n, p-1}-$ $Z_{n, p-1} \in \operatorname{Im}_{\operatorname{Inf}_{\mathbb{M}_{n}}^{W}}^{W}$

(ii) if $n>1$ then $\operatorname{Res}_{{ }^{\prime} \Gamma_{n-1}}^{\mathbb{M}_{n}}\left(X_{n, i}\right)-x Y_{n-1, i} \in \operatorname{Im} \operatorname{Inf}_{{ }^{5} \Gamma_{n-1}}^{\Gamma_{n-1} / Z}$;

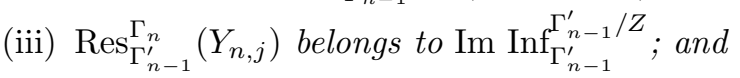

(iv) $x Y_{n, i} \notin(y)$; furthermore, there exists no element $\xi \in H^{*}\left(\Gamma_{n}\right)$ satisfying $x Y_{n, i}=y \xi \bmod \operatorname{Im} \operatorname{Inf}_{\Gamma_{n}}^{V}$.

Proof. (i) and (ii) follow by considering the restriction in spectral sequences and by the structures of $E_{2 p+1}\left(\mathbb{M}_{n}\right)$ and $E_{2 p+1}\left(\Gamma_{n}\right)$ given in Theorem 1 (ii) and Proposition 2 . 
(iii) Set $T_{n, i}=\operatorname{Res}_{\Gamma_{n-1}^{\prime}}^{\Gamma_{n}}\left(Y_{n, i}\right)$. Since $y_{2 n-1} Y_{n, i} \in \operatorname{Im} \operatorname{Inf}_{\Gamma_{n}}^{V}$, it follows that

$$
y_{2 n-1} T_{n, i}=\operatorname{Res}_{\Gamma_{n-1}^{\prime}}^{\Gamma_{n}}\left(y_{2 n-1} Y_{n, i}\right)
$$

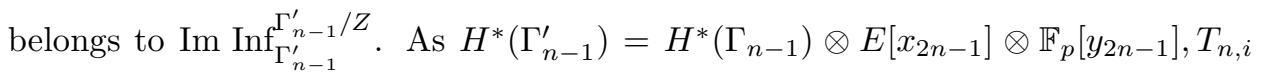
also belongs to $\operatorname{Im} \operatorname{Inf}_{\Gamma_{n-1}^{\prime}}^{\Gamma_{n-1}^{\prime} / Z}$.

(iv) Assume that there exists $\xi \in H^{2 n+1}\left(\Gamma_{n}\right)$ such that

$$
y \xi=x Y_{n, 1} \bmod \operatorname{Im} \operatorname{Inf}_{\Gamma_{n}}^{V} .
$$

By Proposition 3, $\xi \in \operatorname{Im}_{\operatorname{Inf}_{\Gamma_{n}}}^{V}$. So $y \xi \in \operatorname{Im}_{\operatorname{Inf}_{\Gamma_{n}}}^{V}$. Hence $x Y_{n, 1} \in \operatorname{Im}_{\operatorname{Inf}_{\Gamma_{n}}}^{V}$, a contradiction.

Assume inductively that (iv) holds for $i-1$. For $i \geq 2$, we will prove in Lemmas 10, 11, 16 and 17 that $\operatorname{tr}_{\Gamma_{n+1}}^{\Gamma_{n}^{\prime}}\left(Y_{n, i}\right)=\lambda_{i} Y_{n+1, i-1} \bmod \operatorname{Im}_{\operatorname{Inf}_{\Gamma_{n+1}}}^{V}$ with $0 \neq \lambda_{i} \in \mathbb{F}_{p}$. Let $\phi$ be the element of $\operatorname{Im} \operatorname{Inf}_{\Gamma_{n+1}}^{V}$ satisfying $\operatorname{tr}_{\Gamma_{n+1}}^{\Gamma_{n}^{\prime}}\left(Y_{n, i}\right)=\lambda_{i} Y_{n+1, i-1}+\phi$. Suppose that $x Y_{n, i}+\eta=y \xi$, with $\xi \in H^{*}\left(\Gamma_{n}\right)$ and $\eta \in \operatorname{Im}_{\operatorname{Inf}_{\Gamma_{n}}^{V}}^{V}$. So

$$
\begin{aligned}
\lambda_{i} x Y_{n+1, i-1}+x \phi & =x \operatorname{tr}_{\Gamma_{n+1}}^{\Gamma_{n}^{\prime}}\left(Y_{n, i}\right) \\
& =\operatorname{tr}_{\Gamma_{n+1}^{\Gamma_{n}}}^{\Gamma_{n}}\left(x Y_{n, i}+\eta\right) \quad \text { since } \operatorname{tr}_{\Gamma_{n+1}}^{\Gamma_{n}^{\prime}}(\eta)=0 \\
& =\operatorname{tr}_{\Gamma_{n+1}^{\prime}}^{\Gamma_{n}^{\prime}}(y \xi) \\
& =y \operatorname{tr}_{\Gamma_{n+1}}^{\Gamma_{n}^{\prime}}(\xi) .
\end{aligned}
$$

Hence $\lambda_{i} x Y_{n+1, i-1}=y \operatorname{tr}_{\Gamma_{n+1}}^{\Gamma_{n}^{\prime}}(\xi)-x \phi$, which contradicts the inductive hypothesis. (iv) is then proved.

The lemma follows.

Further properties of $X_{n, i}$ and $Y_{n, i}$ are given by the following lemmas. The first one follows from Theorem 1, Proposition 2 and [14, Theorem 1.1].

Lemma 8. $\operatorname{tr}_{\mathbb{M}_{n}^{\prime}}^{\mathbb{M}_{n-1}^{\prime}}\left(X_{n-1, i}\right)$ (resp. $\operatorname{tr}_{\Gamma_{n}}^{\Gamma_{n-1}^{\prime}}\left(Y_{n-1, i}\right)$ ) represents an element of $E_{\infty}^{*, 2 j}\left(\mathbb{M}_{n}\right)$ (resp. $\left.E_{\infty}^{*, 2 j}\left(\Gamma_{n}\right)\right)$, with $j<i$.

Lemma 9. For $2 \leq i \leq p-1$ we have $\operatorname{tr}_{\Gamma_{1}}^{\Gamma_{0}^{\prime}}\left(Y_{0, i}\right)=\lambda_{i} Y_{1, i-1} \bmod \operatorname{Im}_{\operatorname{Inf}_{\Gamma_{1}}^{V}}^{V}$, with $0 \neq \lambda_{i} \in \mathbb{F}_{p}$.

Proof. Consider the commutative diagram

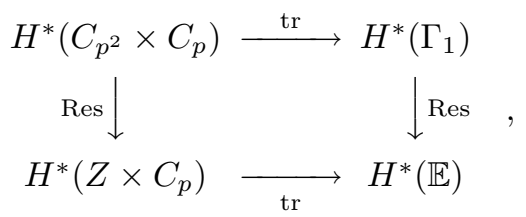

We have $\operatorname{Res}_{\mathbb{E}}^{\Gamma_{1}} \operatorname{tr}_{\Gamma_{1}}^{\Gamma_{0}^{\prime}}\left(Y_{0, i}\right)=\operatorname{tr}_{\mathbb{E}}^{Z \times C_{p}}\left(v^{i}\right)$. Following [8] (see also [16]), $\operatorname{tr}_{\mathbb{E}}^{Z \times C_{p}}\left(v^{i}\right)$ is a non-zero element of $H^{*}(\mathbb{E}) \backslash \operatorname{Im} \operatorname{Inf}_{\mathbb{E}}^{\mathbb{E} / Z}$. So, by Theorem $1, \operatorname{tr}_{\Gamma_{1}^{\prime}}^{\Gamma_{0}^{\prime}}\left(Y_{0, i}\right)$ represents an element of the form $\lambda_{i} x_{1} x_{2} \otimes v^{i-1} \in E_{\infty}^{2,2(i-1)}\left(\Gamma_{1}\right)$, with $0 \neq \lambda_{i} \in \mathbb{F}_{p}$. The lemma follows.

In the following two lemmas, $p$ is assumed to be greater than 3 . 
Lemma 10. For $2 \leq i \leq p-2$,

$$
\operatorname{tr}_{\mathbb{M}_{2}}^{\mathbb{M}_{1}^{\prime}}\left(X_{1, i}\right)=\lambda_{i} X_{2, i-1} \bmod \operatorname{Im} \operatorname{Inf}_{\mathbb{M}_{2}}^{W}
$$

and

$$
\operatorname{tr}_{\Gamma_{2}}^{\Gamma_{1}^{\prime}}\left(Y_{1, i}\right)=\lambda_{i} Y_{2, i-1} \bmod \operatorname{Im} \operatorname{Inf}_{\Gamma_{2}}^{V},
$$

with $\lambda_{i}$ given in Lemma 9.

Proof. Set $Z_{i}=\operatorname{Res}_{\Gamma_{1}}^{\mathbb{M}_{2}} \operatorname{tr}_{\mathbb{M}_{2}}^{\mathbb{M}_{1}^{\prime}}\left(X_{1, i}\right)$. By the double coset formula and by Lemma 7 (ii), we have

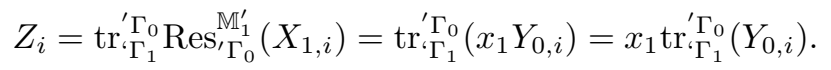

By Lemma $9, Z_{i}$ represents

$$
\lambda_{i} x_{1} x_{3} x_{4} \otimes v^{i-1} \in E_{\infty}^{3,2(i-1)}\left({ }^{6} \Gamma_{1}\right) .
$$

By Lemma 8 and Proposition 2, this means that $\operatorname{tr}_{\mathbb{M}_{2}}^{\mathbb{M}_{1}^{\prime}}\left(X_{1, i}\right)$ represents

$$
\lambda_{i} x_{1} x_{3} x_{4} \otimes v^{i-1} \in E_{\infty}^{3,2(i-1)}\left(\mathbb{M}_{2}\right) .
$$

The first part of the lemma follows from the definition of $X_{2, i-1}$.

On the other hand, by setting $Y_{i}=\operatorname{Res}_{\mathbb{M}_{2}}^{\Gamma_{2}} \operatorname{tr}_{\Gamma_{2}}^{\Gamma_{1}^{\prime}}\left(Y_{1, i}\right)$, by the double coset formula, we have

$$
\begin{aligned}
Y_{i} & =\operatorname{tr}_{\mathbb{M}_{2}}^{\mathbb{M}_{1}^{\prime}} \operatorname{Res}_{\mathbb{M}_{1}^{\prime}}^{\Gamma_{1}^{\prime}}\left(Y_{1, i}\right) \\
& =\operatorname{tr}_{\mathbb{M}_{2}}^{\mathbb{M}_{1}^{\prime}}\left(-x_{2} X_{1, i}\right) \quad \text { by Lemma } 7 \text { (i) } \\
& =-x_{2} \operatorname{tr}_{\mathbb{M}_{2}^{\prime}}^{\mathbb{M}_{1}^{\prime}}\left(X_{1, i}\right) .
\end{aligned}
$$

As shown above, $\operatorname{tr}_{\mathbb{M}_{2}}^{\mathbb{M}_{1}^{\prime}}\left(X_{1, i}\right)$ represents $\lambda_{i} x_{1} x_{3} x_{4} \otimes v^{i-1} \in E_{\infty}^{3,2(i-1)}\left(\mathbb{M}_{2}\right)$, so $Y_{i}$ represents $\lambda_{i} x_{1} x_{2} x_{3} x_{4} \otimes v^{i-1} \in E_{\infty}^{4,2(i-1)}\left(\mathbb{M}_{2}\right)$. By Lemma 8 and Theorem 1 (ii), this means that $\operatorname{tr}_{\Gamma_{2}}^{\Gamma_{1}^{\prime}}\left(Y_{1, i}\right)$ represents $\lambda_{i} x_{1} x_{2} x_{3} x_{4} \otimes v^{i-1} \in E_{\infty}^{4,2(i-1)}\left(\Gamma_{2}\right)$. The last part follows from the definition of $Y_{2, i-1}$. The lemma is proved.

In general, we have

Lemma 11. For $2 \leq i \leq p-2$ and $n \geq 2$,

$$
\operatorname{tr}_{\mathbb{M}_{n}^{\prime}}^{\mathbb{M}_{n-1}^{\prime}}\left(X_{n-1, i}\right)=\lambda_{i} X_{n, i-1} \bmod \operatorname{Im} \operatorname{Inf}_{\mathbb{M}_{n}}^{W}
$$

and

$$
\operatorname{tr}_{\Gamma_{n}}^{\Gamma_{n-1}^{\prime}}\left(Y_{n-1, i}\right)=\lambda_{i} Y_{n, i-1} \bmod \operatorname{Im} \operatorname{Inf}_{\Gamma_{n}}^{V},
$$

with $\lambda_{i}$ given in Lemma 9.

Proof. We argue by induction on $n$. The case $n=2$ follows from the above lemma. Assume that the lemma holds for $n-1$. Set $Z_{i}=\operatorname{Res}_{{ }^{\prime} \Gamma_{n-1}}^{\mathbb{M}_{n}} \operatorname{tr}_{\mathbb{M}_{n}}^{\mathbb{M}_{n-1}^{\prime}}\left(X_{n-1, i}\right)$. By the double coset formula, we have

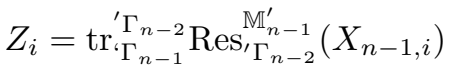

$$
\begin{aligned}
& =\operatorname{tr}{ }^{\prime} \Gamma_{n-2} \Gamma_{n-1}\left(x Y_{n-2, i}\right) \text { by Lemma } 7 \text { (ii) } \\
& =x \operatorname{tr}^{\prime}{ }^{\prime} \Gamma_{n-2}\left(Y_{n-2, i}\right) \text {. }
\end{aligned}
$$




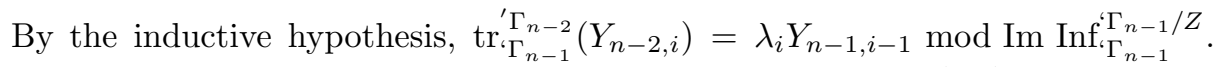
So $Z_{i}$ and $\lambda_{i} x Y_{n-1, i-1}$ represent the same element of $E_{\infty}^{2 n+1,2(i-1)}\left({ }^{\prime} \Gamma_{n-1}\right)$. The first part follows from Lemma 8 and Proposition 2.

Finally, by setting $Y_{i}=\operatorname{Res}_{\mathbb{M}_{n}}^{\Gamma_{n}} \operatorname{tr}_{\Gamma_{n}}^{\Gamma_{n-1}^{\prime}}\left(Y_{n-1, i}\right)$, we have

$$
\begin{aligned}
Y_{i} & =\operatorname{tr}_{\mathbb{M}_{n}}^{\mathbb{M}_{n-1}^{\prime}} \operatorname{Res}_{\mathbb{M}_{n-1}^{\prime}}^{\Gamma_{n-1}^{\prime}}\left(Y_{n-1, i}\right) \\
& =\operatorname{tr}_{\mathbb{M}_{n-1}}^{\mathbb{M}_{n}^{\prime}}\left(-x_{2} X_{n-1, i}\right) \quad \text { by Lemma } 7 \text { (i) } \\
& =-x_{2} \operatorname{tr}_{\mathbb{M}_{n}^{\prime}}^{\mathbb{M}_{n-1}^{\prime}}\left(X_{n-1, i}\right) .
\end{aligned}
$$

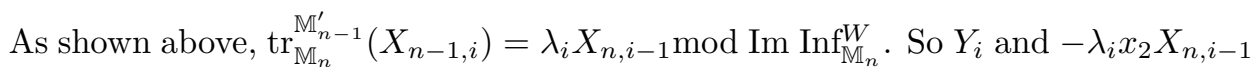
represent the same element of $E_{\infty}^{2 n, 2(i-1)}\left(\mathbb{M}_{n}\right)$. The last part follows from Lemma 8 and Theorem 1 (ii). The lemma is proved.

We now calculate $\operatorname{tr}_{\Gamma_{n}}^{\Gamma_{n-1}^{\prime}}\left(Y_{n-1,1}\right)$. In so doing, let us recall the determination of the transfer map on bar cochain levels. Let $L, K$ be subgroups of $\Gamma_{n}$ with $Z \subset L \subset K$ and let $D=\{d\}$ be the set of cosets of $L$ in $K$. For each $d$, specify a representative $\bar{d}$ of $d$ such that $\bar{L}=1$ and $\bar{d} \overline{d^{\prime}} \overline{d d^{\prime}}-1 \in Z$. The transfer map $\tilde{\operatorname{tr}}_{K}^{L}: C^{*}(L) \rightarrow C^{*}(K)$ is determined in [20] as follows:

$$
\begin{aligned}
\tilde{\operatorname{tr}}_{K}^{L} f\left(\cdot{ }_{L}\right) & =\sum_{d \in D} f\left(\cdot \cdot_{K}\right) \\
\tilde{\operatorname{tr}}_{K}^{L} f\left(\ell_{1}, \ldots, \ell_{n}\right) & =\sum_{d \in D} f\left(\bar{d} \ell_{1} \overline{d \ell_{1}}-1, \ldots, \overline{d \ell_{1} \ldots \ell_{n-1}} \ell_{n} \frac{\overline{d \ell_{1} \ldots \ell_{n-1} \ell_{n}}}{-1}\right)
\end{aligned}
$$

for $f \in C^{*}(L), \ell_{i} \in K$.

Some properties of $\tilde{\operatorname{tr}}_{K}^{L}$ were given in [14]. Note that, if $L$ is a direct factor of $K$, then $\tilde{\operatorname{tr}}_{K}^{L}$ is the zero map. Furthermore, if $M$ is also a subgroup of $\Gamma_{n}$ containing $Z$, we can choose representatives of the cosets of $M$ in $K M$, and those of $K \cap M$ in $K$, so that the double coset formula

$$
\operatorname{Res}_{K}^{K M} \tilde{\operatorname{tr}}_{K M}^{M}=\tilde{\operatorname{tr}}_{K}^{K \cap M} \operatorname{Res}_{K \cap M}^{M}
$$

holds at the cochain level.

Since $v \in E_{2}\left(\Gamma_{n-1}^{\prime}\right)$ is transgressive, there exists a 2-cochain $\tilde{v}$ of $\Gamma_{n-1}^{\prime}$ satisfying $\left.\tilde{v}\right|_{Z}=v, \delta \tilde{v}=\beta z_{n-1}$ (see e.g. [15] for a determination of such a cochain). It follows

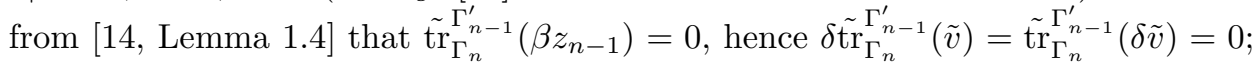
in other words, $\tilde{\operatorname{tr}}_{\Gamma_{n}}^{\Gamma_{n-1}^{\prime}}(\tilde{v})$ is a 2-cocycle of $\Gamma_{n}$. Set $\bar{v}=\left[\tilde{\operatorname{tr}}_{\Gamma_{n}}^{\Gamma_{n-1}^{\prime}}(\tilde{v})\right] \in H^{2}\left(\Gamma_{n}\right)$ and let $\tilde{e}, \tilde{a}_{1}, \ldots, \tilde{a}_{2 n}$ be elements of $\Gamma_{n}$ satisfying $\tilde{e} Z=e, \tilde{a}_{i} Z=a_{i}$ (recall that $e, a_{1}, \ldots, a_{2 n}$ was defined in Section 2 as a basis of $V$ of which the dual is $\left.x, x_{1}, \ldots, x_{2 n}\right)$. We have

Lemma 12. $\bar{v}=-x_{2 n-1} x_{2 n}$.

Proof. Write

$$
\bar{v}=\sum_{1 \leq i \leq 2 n} \mu_{i} x x_{i}+\sum_{1 \leq i<j \leq 2 n} \mu_{i j} x_{i} x_{j}+\sum_{1 \leq i \leq 2 n} \nu_{i} y_{i}
$$

with $\mu_{i}, \mu_{i j}, \nu_{i} \in \mathbb{F}_{p}$ (note that, in $H^{2}\left(\Gamma_{n}\right), y=-\left(x_{1} x_{2}+\cdots+x_{2 n-1} x_{2 n}\right)$ ). Consider the double coset formula (5) with $M=\Gamma_{n-1}^{\prime}$ and $K M=\Gamma_{n}$ (this means that 
$\left.\tilde{a}_{2 n} \in K\right)$. For $K=\left\langle\tilde{e}, \tilde{a}_{i}, \tilde{a}_{j}, \tilde{a}_{2 n}\right\rangle$ with $1 \leq i, j \leq 2 n-2$, as $\tilde{a}_{2 n}$ commutes with every element of $K \cap M$, we have $\tilde{\operatorname{tr}}_{K}^{K \cap \bar{M}}=0$, so $\mu_{i}=\mu_{2 n}=\nu_{i}=\nu_{2 n}=$ $\mu_{i j}=\mu_{i 2 n}=0$. For $K=\left\langle\tilde{e}, \tilde{a}_{i}, a_{2 n-1}, \tilde{a}_{2 n}\right\rangle$ with $1 \leq i \leq 2 n-2$, we have $K \cong \Gamma_{1} \times C_{p}, K \cap M=C_{p^{2}} \times C_{p}^{2}$ and $\operatorname{Res}_{K \cap M}^{M}(\tilde{v})=w$; by a direct verification, we can show that $\operatorname{tr}_{K}^{K \cap M}(w)=y$, therefore $\left[\tilde{\operatorname{tr}}_{K}^{K \cap M}(w)\right]=y=-x_{2 n-1} x_{2 n}$, so $\mu_{2 n-1}=\mu_{i 2 n-1}=\nu_{2 n-1}=0$ and $\mu_{2 n-12 n}=-1$. The lemma follows.

Lemma 13. For $n \geq 1, \operatorname{tr}_{\Gamma_{n}}^{\Gamma_{n-1}^{\prime}}\left(Y_{n-1,1}\right)=-X_{n}$; hence $\operatorname{Res}_{\mathbb{E}_{n}}^{\Gamma_{n}} \operatorname{tr}_{\Gamma_{n}}^{\Gamma_{n-1}^{\prime}}\left(Y_{n-1,1}\right)=0$.

Proof. A cocycle representing $Y_{n-1,1}$ can be chosen as follows. Since $x_{1} x_{2} \ldots x_{2 n-3}$. $x_{2 n-2} \cdot \beta z_{n-1}=0$ in $H^{*}\left(\Gamma_{n-1}^{\prime} / Z\right)$, there exists a cochain $f$ of $\Gamma_{n-1}^{\prime} / Z$ (considered as a cochain of $\Gamma_{n-1}^{\prime}$ via the inflation map on cochains) satisfying $\delta f=x_{1} x_{2} \ldots x_{2 n-3}$. $x_{2 n-2} \cdot \beta z_{n-1}$. Furthermore, it follows from the definition of $\tilde{v}$ that

$$
\delta\left(x_{1} x_{2} \ldots x_{2 n-3} x_{2 n-2} \cdot \tilde{v}\right)=x_{1} x_{2} \ldots x_{2 n-3} x_{2 n-2} \cdot \beta z_{n-1} ;
$$

hence $\delta\left(x_{1} x_{2} \ldots x_{2 n-3} x_{2 n-2} \cdot \tilde{v}-f\right)=0$. Clearly $g=x_{1} x_{2} \ldots x_{2 n-3} x_{2 n-2} \cdot \tilde{v}-f$ is a cocycle representing $X_{n} \otimes v \in E_{\infty}\left(\Gamma_{n-1}^{\prime}\right)$. Hence

$$
Y_{n-1,1}-[g] \in \operatorname{Im} \operatorname{Inf}_{\Gamma_{n-1}}^{\Gamma_{n-1}^{\prime} / Z}
$$

which implies that $\operatorname{tr}_{\Gamma_{n}}^{\Gamma_{n-1}^{\prime}}\left(Y_{n-1,1}\right)$ is represented by $\tilde{\operatorname{tr}}_{\Gamma_{n}}^{\Gamma_{n-1}^{\prime}}(g)$. By [14, Lemma 1.4], $\tilde{\operatorname{tr}}_{\Gamma_{n}}^{\Gamma_{n-1}^{\prime}}(g)=x_{1} x_{2} \ldots x_{2 n-3} x_{2 n-2} \cdot \tilde{\operatorname{tr}}_{\Gamma_{n}^{n-1}}^{\Gamma_{n}^{\prime}}(\tilde{v})$. So $\left[\tilde{\operatorname{tr}}_{\Gamma_{n}}^{\Gamma_{n-1}^{\prime}}(g)\right]=x_{1} x_{2} \ldots x_{2 n-3} x_{2 n-2} \cdot \bar{v}$. The lemma now follows from Lemma 12.

Arguing as in the above proof, we can also choose a cocycle representing $X_{n-1} \otimes$ $v^{p}$ (which is non-zero in $E_{\infty}\left(\Gamma_{n-1}^{\prime}\right)$, by Theorem 1 , Propositions 5 and 7 ), as follows. As $v^{p} \in E_{2}\left(\Gamma_{n-1}^{\prime}\right)$ is transgressive and $d_{2 p+1}\left(v^{p}\right)=\mathcal{P}^{1} \beta z_{n-1}$, there exists a cochain $\widetilde{v^{p}}$ of $\Gamma_{n-1}^{\prime}$ such that $\left.\widetilde{v^{p}}\right|_{Z}=v^{p}$, and $\delta \widetilde{v^{p}}=\mathcal{P}^{1} \beta z_{n-1}$. Let $h$ be a cochain of $\Gamma_{n-1}^{\prime} / Z$ satisfying $\delta h=\mathcal{P}^{1} \beta z_{n-1} \cdot X_{n-1}$. We have

Lemma 14. $k=k_{n}=\widetilde{v^{p}} \cdot x_{1} x_{2} \ldots x_{2 n-3} x_{2 n-2}-h$ is a cocycle representing $X_{n-1} \otimes$ $v^{p}$ and $\operatorname{Res}_{\mathbb{E}_{n}}^{\Gamma_{n}} \operatorname{tr}_{\Gamma_{n}}^{\Gamma_{n-1}^{\prime}}([k])=0$.

Proof. It follows from the definitions of $\widetilde{v^{p}}$ and $h$ that $k$ is a cocycle representing $X_{n-1} \otimes v^{p}$. Set $X=\operatorname{Res}_{\mathbb{E}_{n}}^{\Gamma_{n}} \operatorname{tr}_{\Gamma_{n}}^{\Gamma_{n-1}^{\prime}}([k])$; then $X=\left[\tilde{\operatorname{tr}}_{\mathbb{E}_{n}}^{\mathbb{E}_{n-1} \times C_{p}} \operatorname{Res}_{\mathbb{E}_{n-1} \times C_{p}}^{\Gamma_{n-1}^{\prime}}(k)\right]$ by the double coset formula. Denote also by $\widetilde{v^{p}}$ (resp. $h$ ) the restriction of the cochain $\widetilde{v^{p}}($ resp. $h)$ to $\mathbb{E}_{n-1} \times C_{p}$. By [14, Lemma 1.4], $\tilde{\operatorname{tr}}_{\mathbb{E}_{n}}^{\mathbb{E}_{n-1} \times C_{p}}(h)=0$; hence $X=$ $\left[\tilde{\operatorname{tr}}_{\mathbb{E}_{n}}^{\mathbb{E}_{n-1} \times C_{p}}\left(\widetilde{v^{p}} \cdot x_{1} x_{2} \ldots x_{2 n-3} x_{2 n-2}\right)\right]$. Note that, in $H^{*}\left(\mathbb{E}_{n-1} \times C_{p}\right)$ we have $X_{n-1}=$ $X_{n-2}\left(x_{1} x_{2}+\cdots+x_{2 n-3} x_{2 n-2}\right)$ and $x_{1} x_{2}+\cdots+x_{2 n-3} x_{2 n-2}=0$, so there exist cochains $c$ of $\left(\mathbb{E}_{n-1} \times C_{p}\right) / Z$ and $b$ of $\mathbb{E}_{n-1} \times C_{p}$ satisfying

$$
\begin{aligned}
\delta b & =x_{1} x_{2}+\cdots+x_{2 n-3} x_{2 n-2}, \\
x_{1} x_{2} \ldots x_{2 n-3} x_{2 n-2} & =x_{1} x_{2} \ldots x_{2 n-5} x_{2 n-4} \cdot \delta b+\delta c .
\end{aligned}
$$

Hence

$$
\begin{aligned}
\widetilde{v^{p}} \cdot x_{1} x_{2} \ldots x_{2 n-3} x_{2 n-2} & =\widetilde{v^{p}} \cdot x_{1} x_{2} \ldots x_{2 n-5} x_{2 n-4} \cdot \delta b+\widetilde{v^{p}} \cdot \delta c \\
& =-\delta \widetilde{v^{p}} \cdot x_{1} x_{2} \ldots x_{2 n-5} x_{2 n-4} \cdot b-\delta \widetilde{v^{p}} \cdot c \bmod \operatorname{Im} \delta
\end{aligned}
$$


So $X=-\left[\tilde{\operatorname{tr}}_{\mathbb{E}_{n-1} \times C_{p}}^{\mathbb{E}_{n}}\left(\delta \widetilde{v^{p}} \cdot x_{1} x_{2} \ldots x_{2 n-5} x_{2 n-4} \cdot b+\delta \widetilde{v^{p}} \cdot c\right)\right]$. Following [14, Lemma

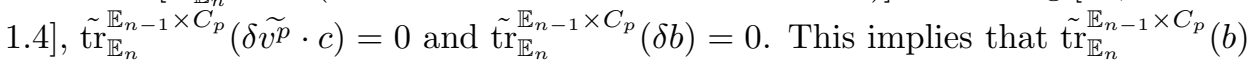
is a cocycle of $\mathbb{E}_{n}$ and

$$
X=-\mathcal{P}^{1} \beta z_{n-1} \cdot X_{n-2} \cdot\left[\tilde{\operatorname{tr}}_{\mathbb{E}_{n}}^{\mathbb{E}_{n-1} \times C_{p}}(b)\right] .
$$

Arguing as in the proof of Lemma 12, we can show that $\left[\tilde{\operatorname{tr}}_{\mathbb{E}_{n-1}}^{\mathbb{E}_{n} \times C_{p}}(b)\right]=0$. Hence $X=0$. The lemma follows.

With some abuse of notation, we also denote by $\tilde{v}$ (resp. $\widetilde{v^{p}}$ ) the restriction of $\tilde{v}$ (resp. $\left.\widetilde{v^{p}}\right)$ to $\mathbb{M}_{n-1}^{\prime}$. So $\delta(\tilde{v})=\beta z_{n-1}^{\prime}$ and $\delta\left(\widetilde{v^{p}}\right)=\mathcal{P}^{1} \beta z_{n-1}^{\prime}$ in $C^{*}\left(\mathbb{M}_{n-1}^{\prime}\right)$. Let $\tilde{u}$ be a 1-cochain of $\mathbb{M}_{n-1}^{\prime}$ satisfying $\delta(\tilde{u})=z_{n-1}^{\prime}$. It follows from the proof of Proposition 8 that there exists a cochain $d$ of $\mathbb{M}_{n-1}^{\prime} / Z$ such that

$$
\begin{aligned}
\delta d & =x_{3} x_{4} \ldots x_{2 n-3} x_{2 n-2}\left(\mathcal{P}^{1} \beta z_{n-1}^{\prime}-x_{2} z^{\prime p}{ }_{n-1}-y_{2}^{p-1} \beta z_{n-1}^{\prime}+y_{2}^{p-1} x_{2} z_{n-1}^{\prime}\right) \\
& =\delta\left(x_{3} x_{4} \ldots x_{2 n-3} x_{2 n-2}\left(\widetilde{v^{p}}+x_{2} z_{n-1}^{\prime p-1} \tilde{u}-y_{2}^{p-1} \tilde{v}-y_{2}^{p-1} x_{2} \tilde{u}\right)\right) .
\end{aligned}
$$

So, for $n \geq 3, q=x_{3} x_{4} \ldots x_{2 n-3} x_{2 n-2}\left(\widetilde{v^{p}}+x_{2}{z^{\prime}}_{n-1}^{p-1} \tilde{u}-y_{2}^{p-1} \tilde{v}-y_{2}^{p-1} x_{2} \tilde{u}\right)-d$ is a cocycle of $\mathbb{M}_{n-1}^{\prime}$ representing $Z_{n-1, p-1}$. We have

Lemma 15. For $n \geq 3$,

$$
\operatorname{tr}_{\mathbb{M}_{n}^{\prime}}^{\mathbb{M}_{n-1}^{\prime}}\left(Z_{n-1, p-1}\right) \in \operatorname{ImInf}_{\mathbb{M}_{n}}^{W}
$$

Proof. It follows that $\operatorname{tr}_{\mathbb{M}_{n-1}^{\prime}}^{\mathbb{M}_{n}^{\prime}}\left(Z_{n-1, p-1}\right)=\left[\tilde{\tilde{t r}_{\mathbb{M}_{n}}} \mathbb{M}_{n-1}^{\prime}(q)\right]$. By [14, Lemma 1.4],

$$
\begin{aligned}
\operatorname{tr}_{\mathbb{M}_{n}}^{\mathbb{M}_{n-1}^{\prime}}\left(Z_{n-1, p-1}\right)=x_{3} x_{4} \ldots x_{2 n-3} x_{2 n-2}\left(\left[\tilde{\operatorname{tr}}_{\mathbb{M}_{n-1}^{\prime}}\left(\widetilde{\mathbb{v}^{p}}\right)\right]+x_{2} z_{n-1}^{\prime p-1}\left[\tilde{\operatorname{tr}}_{\mathbb{M}_{n}^{\prime}}^{\prime}(\tilde{u})\right]\right. \\
\left.-y_{2}^{p-1}\left[\tilde{\operatorname{tr}}_{\mathbb{M}_{n}^{\prime}}^{\prime}(\tilde{v})\right]-y_{2}^{p-1} x_{2}\left[\tilde{\mathbb{t r}_{\mathbb{M}_{n-1}}} \mathbb{M}_{n}^{\prime}(\tilde{u})\right]\right)
\end{aligned}
$$

(note that $\tilde{\mathrm{tr}_{\mathbb{M}_{n}}} \mathbb{M}_{n-1}^{\prime}$ maps each of $\widetilde{v^{p}}, \tilde{u}, \tilde{v}$ to a cocycle). Since each of $\tilde{\operatorname{tr}}_{\mathbb{M}_{n}^{\prime}} \mathbb{M}_{n}^{\prime}\left(\widetilde{v^{p}}\right)$, $\tilde{\operatorname{tr}_{\mathbb{M}_{n}}} \mathbb{M}_{n-1}^{\prime}(\tilde{v}), \tilde{\operatorname{tr}_{\mathbb{M}_{n}} \mathbb{M}_{n-1}^{n}}(\tilde{u})$ is of degree $\leq 2 p$, it follows from the structure of $E_{2 p+1}\left(\mathbb{M}_{n}\right)$ that $\left[\tilde{\operatorname{tr}}_{\mathbb{M}_{n}^{\prime}}^{\mathbb{M}_{n}^{\prime}}(\tilde{v})\right],\left[\tilde{\operatorname{tr}}_{\mathbb{M}_{n-1}^{\prime}}^{\prime}(\tilde{u})\right]$ and the cup-product of $x_{3} x_{4} \ldots x_{2 n-3} x_{2 n-2}$ with $\left[\tilde{\operatorname{tr}}_{\mathbb{M}_{n}}^{\mathbb{M}_{n-1}^{\prime}}\left(\widetilde{v^{p}}\right)\right]$ belong to $\operatorname{Im} \operatorname{Inf}_{\mathbb{M}_{n}}^{W}$. The lemma follows.

Lemma 16. There exists a non-zero $\lambda \in \mathbb{F}_{p}$ such that $\operatorname{tr}_{\Gamma_{2}}^{\Gamma_{1}^{\prime}}\left(Y_{1, p-1}\right)-\lambda Y_{2, p-2} \in$ $\operatorname{Im} \operatorname{Inf}_{\Gamma_{2}}^{V}$.

Proof. Set $K=\operatorname{Ker} x_{2} \cap \operatorname{Ker}\left(x-x_{4}\right) \subset \Gamma_{2}$ and $X=\operatorname{tr}_{\Gamma_{2}}^{\Gamma_{1}^{\prime}}\left(Y_{1, p-1}\right)$. So $K \cong \mathbb{M} \times$ $C_{p}, K \cap \Gamma_{1}^{\prime} \cong C_{p}^{3}$ and $\operatorname{Res}_{K}^{\Gamma_{2}}(X)=\operatorname{tr}_{K}^{\Gamma_{1}^{\prime} \cap K} \operatorname{Res}_{\Gamma_{1}^{\prime} \cap K}^{\Gamma_{1}^{\prime}}\left(Y_{1, p-1}\right)$. As $\operatorname{Res}_{\Gamma_{1}^{\prime} \cap K}^{\Gamma_{1}^{\prime}}\left(Y_{1, p-1}\right)=$ $v^{p}-v y_{1}^{p-1}$, we have

$$
\operatorname{Res}_{K}^{\Gamma_{2}}(X)=\operatorname{tr}_{K}^{\Gamma_{1}^{\prime} \cap K}\left(v^{p}-v y_{1}^{p-1}\right)=\operatorname{tr}_{K}^{\Gamma_{1}^{\prime} \cap K}\left(v^{p}\right)-\operatorname{tr}_{K}^{\Gamma_{1}^{\prime} \cap K}\left(v y_{1}^{p-1}\right) .
$$

A direct verification shows that $\operatorname{tr}_{K}^{\Gamma_{1}^{\prime} \cap K}(v)=y_{4}$, so $\operatorname{Res}_{K}^{\Gamma_{2}}(X)=-y_{4} y_{1}^{p-1} \neq 0$. Hence $X \neq 0$.

Suppose that $X \in \operatorname{Im}_{\operatorname{Inf}_{\Gamma_{2}}}^{V}$. Since $y_{4} X=0, y_{4} X$ must belong to $\left(z, \eta_{1}, \eta_{2}, \xi_{1}\right)$. Write

$$
y_{4} X=a z+b \eta_{1}+c \eta_{2}+\mu \xi_{1}
$$


with $a, b, c \in H^{*}(V)$ and $\mu \in \mathbb{F}_{p}$. Multiplying (6) by $x_{1} x_{2} x_{3} x_{4}$ yields $\mu \xi_{1} \in\left(y, y_{4}\right)$. Hence $\mu=0$. Multiplying (6) by $\eta_{2}$ yields $y_{4} X \eta_{2} \in\left(z, \eta_{1}\right)$. So, by [13, Lemma 2.4], $X \eta_{2} \in\left(z, \eta_{1}, X_{2}\right)$. Since $X \eta_{2}$ is of degree $>4$, it follows that $X \eta_{2} \in\left(z, \eta_{1}\right)$. By [13, Lemma 2.14], $X=e y \bmod \left(z, \eta_{1}\right)$ with $e \in H^{2 p-2}(V)$. Write

$$
\text { eyy } y_{4}=a_{1} z+b_{1} \eta_{1}+c_{1} \eta_{2} .
$$

Multiplying (7) by $\eta_{1} \eta_{2}$ yields

$$
\begin{aligned}
e y y_{4} \eta_{1} \eta_{2} & =a_{1} z \eta_{1} \eta_{2} \\
& =a_{1} y \eta_{1} \eta_{2}-a_{1} X_{2} \xi_{1} .
\end{aligned}
$$

So $a_{1} \in\left(y, x_{1}, \ldots, x_{4}\right)$. Therefore $b_{1} \in\left(y, x_{i} x_{j}\right)$ and $c_{1}=0$. By [13 Lemma 2.4], we have $e y \in\left(z, \eta_{1}, X_{2}\right)$. Since $e y$ is of degree $>4$, it follows that $e y \in\left(z, \eta_{1}\right)$. So $X \in\left(z, \eta_{1}\right)$, and hence $X=0$ in $H^{*}\left(\Gamma_{2}\right)$, a contradiction. The lemma follows.

Lemma 17. For $n \geq 3$,

$$
\operatorname{tr}_{\mathbb{M}_{n}}^{\mathbb{M}_{n-1}^{\prime}}\left(X_{n-1, p-1}\right)=\lambda X_{n, p-2} \bmod \operatorname{Im} \operatorname{Inf}_{\mathbb{M}_{n}}^{W}
$$

and

$$
\operatorname{tr}_{\Gamma_{n}}^{\Gamma_{n-1}^{\prime}}\left(Y_{n-1, p-1}\right)=\lambda Y_{n, p-2} \bmod \operatorname{Im} \operatorname{Inf}_{\Gamma_{n}}^{V},
$$

with $\lambda$ given in Lemma 16 .

Proof. Consider the case $n=3$. Set $X=\operatorname{Res}_{{ }^{2}}^{\mathbb{M}_{2}} \operatorname{tr}_{\mathbb{M}_{3}}^{\mathbb{M}_{2}^{\prime}}\left(X_{2, p-1}\right)$. By the double coset formula, we have

$$
\begin{aligned}
X & =\operatorname{tr}^{\prime}{ }^{\prime} \Gamma_{1}{ }_{2} \operatorname{Res}{ }^{\mathbb{M}_{2}^{\prime}}{ }_{\Gamma_{1}}^{\prime}\left(X_{2, p-1}\right) \\
& =\operatorname{tr}^{\prime}{ }^{\prime} \Gamma_{2}\left(x Y_{1, p-1}\right) \quad \text { by Lemma } 7 \text { (ii) } \\
& =x \operatorname{tr}^{\prime}{ }^{\prime} \Gamma_{1}\left(Y_{1, p-1}\right) .
\end{aligned}
$$

It follows from Lemma 16 that $X$ and $\lambda x Y_{2, p-2}$ represent the same element of $E_{\infty}^{5,2(p-2)}\left({ }^{'} \Gamma_{2}\right)$. By Lemma 8 and Proposition 2, it follows that $\operatorname{tr}_{\mathbb{M}_{3}}^{\mathbb{M}_{2}^{\prime}}\left(X_{2, p-1}\right)=$ $\lambda X_{3, p-2} \bmod \operatorname{Im} \operatorname{Inf}_{\mathbb{M}_{3}}^{W}$. Similarly, by setting $Y=\operatorname{Res}_{\mathbb{M}_{3}}^{\Gamma_{3}} \operatorname{tr}_{\Gamma_{3}}^{\Gamma_{2}^{\prime}}\left(Y_{2, p-1}\right)$, we have

$$
\begin{aligned}
Y & =\operatorname{tr}_{\mathbb{M}_{3}^{\prime}}^{\mathbb{M}_{2}^{\prime}} \operatorname{Res}_{\mathbb{M}_{2}^{\prime}}^{\Gamma_{2}^{\prime}}\left(Y_{2, p-1}\right) \\
& =\operatorname{tr}_{\mathbb{M}_{3}^{\prime}}^{\mathbb{M}_{2}^{\prime}}\left(-x_{2} X_{2, p-1}+Z_{2, p-1}\right) \quad \text { by Lemma } 7(\mathrm{i}) \\
& =-x_{2} \operatorname{tr}_{\mathbb{M}_{3}}^{\mathbb{M}_{2}^{\prime}}\left(X_{2, p-1}\right)+\operatorname{tr}_{\mathbb{M}_{3}} \mathbb{M}_{2}^{\prime}\left(Z_{2, p-1}\right) .
\end{aligned}
$$

As shown above, $\operatorname{tr}_{\mathbb{M}_{3}}^{\mathbb{M}_{2}^{\prime}}\left(X_{2, p-1}\right)=\lambda X_{3, p-2} \bmod \operatorname{Im} \operatorname{Inf}_{\mathbb{M}_{3}}^{W}$. So, by Lemma $15, Y$ and $-\lambda x_{2} X_{3, p-2}$ represent the same element of $E_{\infty}^{6,2(p-2)}\left(\mathbb{M}_{3}\right)$. By Lemma 8 and Proposition 2, it follows that $\operatorname{tr}_{\Gamma_{3}}^{\Gamma_{2}^{\prime}}\left(Y_{2, p-1}\right)=\lambda Y_{3, p-2} \bmod \operatorname{Im}_{\operatorname{Inf}_{\Gamma_{3}}}^{V}$.

Assume that the lemma holds for $n-1$. Set $Z=\operatorname{Res}_{{ }^{\prime} \Gamma_{n-1}}^{\mathbb{M}_{n}} \operatorname{tr}_{\mathbb{M}_{n}}^{\mathbb{M}_{n-1}^{\prime}}\left(X_{n-1, p-1}\right)$. By the double coset formula, we have

$$
\begin{aligned}
& Z=\operatorname{tr}^{\prime}{ }^{\prime} \Gamma_{n-2}{ }_{\Gamma_{n-1}} \operatorname{Res}{ }^{\mathbb{M}_{n-1}^{\prime}} \Gamma_{n-2}\left(X_{n-1, p-1}\right) \\
& =\operatorname{tr}^{\prime}{ }^{\prime} \Gamma_{n-2}\left(x Y_{n-2, p-1}\right) \text { by Lemma } 7 \text { (ii) } \\
& =x \operatorname{tr}^{\prime}{ }^{\prime} \Gamma_{n-2}\left(Y_{n-2, p-1}\right) \text {. }
\end{aligned}
$$




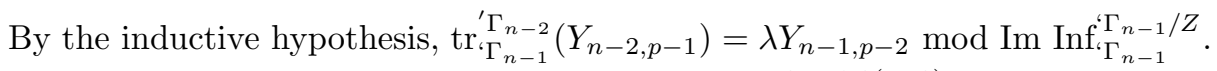
So $Z$ and $\lambda x Y_{n-1, p-2}$ represent the same element of $E_{\infty}^{2 n-1,2(p-2)}\left({ }^{\prime} \Gamma_{n-1}\right)$. The first part follows from Lemma 8 and Proposition 2.

Finally, by setting $Y=\operatorname{Res}_{\mathbb{M}_{n}}^{\Gamma_{n}} \operatorname{tr}_{\Gamma_{n}^{\prime}}^{\Gamma_{n-1}^{\prime}}\left(Y_{n-1, p-1}\right)$, we have

$$
\begin{aligned}
& Y=\operatorname{tr}_{\mathbb{M}_{n}}^{\mathbb{M}_{n-1}^{\prime}} \operatorname{Res}_{\mathbb{M}_{n-1}^{\prime}}^{\Gamma_{n-1}^{\prime}}\left(Y_{n-1, p-1}\right)
\end{aligned}
$$

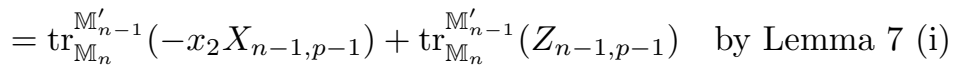

$$
\begin{aligned}
& =-x_{2} \operatorname{tr}_{\mathbb{M}_{n}}^{\mathbb{M}_{n-1}^{\prime}}\left(X_{n-1, p-1}\right)+\operatorname{tr}_{\mathbb{M}_{n}}^{\mathbb{M}_{n-1}^{\prime}}\left(Z_{n-1, p-1}\right) \text {. }
\end{aligned}
$$

As shown above, $\operatorname{tr}_{\mathbb{M}_{n}}^{\mathbb{M}_{n-1}^{\prime}}\left(X_{n-1, p-1}\right)=\lambda X_{n, p-2} \bmod \operatorname{Im} \operatorname{Inf}_{\mathbb{M}_{n}}^{W}$. So, by Lemma 15 , $Y$ and $-\lambda x_{2} X_{n, p-2}$ represent the same element of $E_{\infty}^{2 n, 2(p-2)}\left(\mathbb{M}_{n}\right)$. The last part follows from Lemma 8 and Theorem 1 (ii). The lemma is proved.

Let

$$
\cdots \supset F^{i} C^{*}\left(\Gamma_{n-1}^{\prime}\right) \supset F^{i+1} C^{*}\left(\Gamma_{n-1}^{\prime}\right) \supset \ldots
$$

be the filtration of $C^{*}\left(\Gamma_{n-1}^{\prime}\right)$ introduced by Hochschild and Serre ([7]) corresponding to the central extension $\left(\Gamma_{n-1}^{\prime}\right)$. Let us recall that

$$
F^{i} C^{*}\left(\Gamma_{n-1}^{\prime}\right)= \begin{cases}C^{*}\left(\Gamma_{n-1}^{\prime}\right) & \text { for } i \leq 0, \\ \sum_{m=0}^{\infty} F^{i} C^{m}\left(\Gamma_{n-1}^{\prime}\right) & \text { for } i>0\end{cases}
$$

where $F^{i} C^{m}\left(\Gamma_{n-1}^{\prime}\right)=0$ for $i>m$; and for $0<i \leq m, F^{i} C^{m}\left(\Gamma_{n-1}^{\prime}\right)$ is the group of all $m$-cochains $f$ for which $f\left(g_{1}, \ldots, g_{m}\right)=0$ whenever $m-i+1$ of the arguments $g_{k}$ belong to $Z$. It is clear that the conjugation by $a=a_{2 n}$ on $C^{*}\left(\Gamma_{n-1}^{\prime}\right)$ is compatible with the Hochschild-Serre filtration. We then have the induced conjugation on the Hochschild-Serre spectral sequence $\left\{E_{r}\left(\Gamma_{n-1}^{\prime}\right)\right\}$. As the action of $a$ on $E_{2}^{*, *}\left(\Gamma_{n-1}^{\prime}\right)$ satisfies ${ }^{a} x_{k}=x_{k}, 1 \leq k \leq 2 n-1$, and ${ }^{a} v=v+y_{2 n-1}$, it follows from the structure of $E_{2 p+1}\left(\Gamma_{n-1}^{\prime}\right)$ that $Y_{n-1, i}$ and ${ }^{a} Y_{n-1, i}$ represent the same element of $E_{\infty}\left(\Gamma_{n-1}^{\prime}\right)$. Hence

$$
\begin{aligned}
& Y_{n-1, i}-{ }^{a} Y_{n-1, i}=\sum_{0<j<i} \mu_{j} Y_{n-1, j} y_{2 n-1}^{i-j}+\sum_{0<j<i} \nu_{j} Y_{n-1, j} y_{2 n-1}^{i-j-1} x x_{2 n-1} \\
& \bmod \operatorname{Im} \operatorname{Inf}_{\Gamma_{n-1}^{\prime}}^{\Gamma_{n-1}^{\prime} / Z},
\end{aligned}
$$

with $\mu_{j}, \nu_{j} \in \mathbb{F}_{p}$. We have

Lemma 18. For $n \geq 2$ we have $Y_{n-1,1}-{ }^{a} Y_{n-1,1}=0$.

Proof. Set $K=\operatorname{Ker} x_{2 n-2} \cap \Gamma_{n-1}^{\prime}$. Since the transfer commutes with the conjugation and $\operatorname{Im} \operatorname{Inf}_{\Gamma_{n-1}}^{\Gamma_{n-1}^{\prime} / Z}$ is invariant under the action of $a$, by Lemmas 9, 10, 11, 16 and 17 , we have

$$
Y_{n-1,1}-{ }^{a} Y_{n-1,1}=\operatorname{tr}_{\Gamma_{n-1}^{\prime}}^{K}\left(Y_{n-2,2}-{ }^{a} Y_{n-2,2}\right)
$$

up to a non-zero constant multiple. By Lemma $13, \operatorname{tr}_{\Gamma_{n-1}^{\prime}}^{K}\left(Y_{n-2,1}\right)=-X_{n-1}$; hence $\operatorname{tr}_{\Gamma_{n-1}^{\prime}}^{K}\left(Y_{n-2,1} y_{2 n-3}\right)=0$ and $\operatorname{tr}_{\Gamma_{n-1}^{\prime}}^{K}\left(Y_{n-2,1} x_{2 n-3}\right)=0$ in $H^{*}\left(\Gamma_{n-1}^{\prime}\right)$. The lemma follows from (8) and from the fact that $\operatorname{tr}_{\Gamma_{n-1}^{\prime}}^{K} \operatorname{Inf}_{K}^{K / Z}=0$.

We now have 
Lemma 19. For $n \geq 2$ and $1 \leq i \leq p-1$,

$$
Y_{n-1, i}+{ }^{a} Y_{n-1, i}+\cdots+{ }^{a^{p-1}} Y_{n-1, i}=0
$$

hence

$$
\operatorname{Res}_{\Gamma_{n-1}^{\prime}}^{\Gamma_{n}} \operatorname{tr}_{\Gamma_{n}}^{\Gamma_{n-1}^{\prime}}\left(Y_{n-1, i}\right)=0
$$

Proof. Since $1+a+\cdots+a^{p-1}=(1-a)^{p-1}$, we need prove that ${ }^{(1-a)^{p-1}} Y_{n-1, i}=$ 0 . For $1 \leq k \leq p-1$, by (8) and by Lemma $18,(1-a)^{k} Y_{n-1, k}=0$. Since $\operatorname{Res}_{\Gamma_{n-1}^{\prime}}^{\Gamma_{n}} \operatorname{tr}_{\Gamma_{n}}^{\Gamma_{n-1}^{\prime}}\left(Y_{n-1, i}\right)={ }^{(1-a)^{p-1}} Y_{n-1, i}$, the lemma follows.

For $n \geq 2$ and $1 \leq i \leq p-2$, set $\kappa_{n, i}=\operatorname{Res}_{\mathbb{E}_{n}}^{\Gamma_{n}} \operatorname{tr}_{\Gamma_{n}}^{\Gamma_{n-1}^{\prime}}\left(Y_{n-1, i+1}\right)$. The proof of the theorem is completed by the following fact.

Proposition 9. $0 \neq \kappa_{n, i} \in \operatorname{Ess}\left(\mathbb{E}_{n}\right)$ with $1 \leq i<p-2$ for $p>3$, and $i=1$ for $p=3$.

Proof. It follows from Proposition 1, Lemmas 7 (iv), 9, 11 and 17 that $\kappa_{n, i} \neq 0$ in $H^{*}\left(\mathbb{E}_{n}\right)$. Let $K$ be a maximal subgroup of $\mathbb{E}_{n} . K$ is then of the form $\mathbb{E}_{n-1} \times C_{p}$. Let $L$ be the central product of $K$ and $C_{p^{2}}=\bigcap_{j=1}^{2 n} \operatorname{Ker} x_{j}$. It follows that $L$ is a subgroup of $\Gamma_{n}$ containing $K$ and $L \cong \Gamma_{n-1} \times C_{p}$. Therefore

$$
\begin{aligned}
\operatorname{Res}_{K}^{\mathbb{E}_{n}}\left(\kappa_{n, i}\right) & =\operatorname{Res}_{K}^{\Gamma_{n}} \operatorname{tr}_{\Gamma_{n}}^{\Gamma_{n-1}^{\prime}}\left(Y_{n-1, i+1}\right) \\
& =\operatorname{Res}_{K}^{L} \operatorname{Res}_{L}^{\Gamma_{n}} \operatorname{tr}_{\Gamma_{n}}^{\Gamma_{n-1}^{\prime}}\left(Y_{n-1, i+1}\right) .
\end{aligned}
$$

Hence, if $\Gamma_{n}=\Gamma_{n-1}^{\prime} L$, it follows from the double coset formula that

$$
\operatorname{Res}_{K}^{\mathbb{E}_{n}}\left(\kappa_{n, i}\right)=\operatorname{Res}_{K}^{L} \operatorname{tr}_{L}^{L \cap \Gamma_{n-1}^{\prime}} \operatorname{Res}_{L \cap \Gamma_{n-1}^{\prime}}^{\Gamma_{n-1}^{\prime}}\left(Y_{n-1, i+1}\right) .
$$

By Lemma 1, there exists a non-zero linear combination $\alpha$ of $x_{1}, \ldots, x_{2 n}$ such that $L=\operatorname{Ker} \alpha$. Consider the following cases:

- $\alpha=x_{2 n-1}+\gamma$ with $\gamma$ a linear combination of $x_{1}, \ldots, x_{2 n-2}, x_{2 n}$ : it follows that $\Gamma_{n}=\Gamma_{n-1}^{\prime} L$ and $L \cap \Gamma_{n-1}^{\prime} \cong \Gamma_{n-1}$ is a direct factor of $L$. Hence $\operatorname{tr}_{L}^{L \cap \Gamma_{n-1}^{\prime}}$ is the zero map. We have

$$
\begin{aligned}
\operatorname{Res}_{K}^{\mathbb{E}_{n}}\left(\kappa_{n, i}\right) & =\operatorname{Res}_{K}^{L} \operatorname{tr}_{L}^{L \cap \Gamma_{n-1}^{\prime}} \operatorname{Res}_{L \cap \Gamma_{n-1}^{\prime}}^{\Gamma_{n-1}^{\prime}}\left(Y_{n-1, i+1}\right) \\
& =0 ;
\end{aligned}
$$

- $\alpha=\mu x_{2 n}+\gamma$ with $\gamma$ a non-zero linear combination of $x_{1}, \ldots, x_{2 n-2}$ and $\mu \in \mathbb{F}_{p}$ : it follows that $L \cap \Gamma_{n-1}^{\prime}=H \times\left\langle\tilde{a}_{2 n-1}\right\rangle$ for a subgroup $H$ of $\Gamma_{n-1}$ with $H \cong \Gamma_{n-2}^{\prime}$. If $p>3$, it follows from the proof of Lemma 7 (iii) that $\operatorname{Res}_{L \cap \Gamma_{n-1}^{\prime}}^{\Gamma_{n-1}^{\prime}}\left(Y_{n-1, i+1}\right)$ belongs to the ideal generated by $\operatorname{Im} \operatorname{Inf}_{L \cap \Gamma_{n-1}^{\prime}}^{\left(L \cap \Gamma_{n-1}^{\prime}\right) / Z}$; since

$$
\operatorname{ImInf}_{L \cap \Gamma_{n-1}^{\prime}}^{\left(L \cap \Gamma_{n-1}^{\prime}\right) / Z} \subset \operatorname{Kertr}_{L}^{L \cap \Gamma_{n-1}^{\prime}},
$$

it follows that

$$
\operatorname{Res}_{K}^{\mathbb{E}_{n}}\left(\kappa_{n, i}\right)=0
$$


If $p=3$, by Lemma 14 , there exist $\phi \in H^{2}\left(L \cap \Gamma_{n-1}^{\prime}\right), \psi \in H^{1}\left(L \cap \Gamma_{n-1}^{\prime}\right)$ such that $\operatorname{Res}_{L \cap \Gamma_{n-1}^{\prime}}^{\Gamma_{n-1}^{\prime}}\left(Y_{n-1,2}\right)$ is a linear combination of

$$
\left[k_{n-1}\right], \quad Y_{n-2,1} \cdot \phi, \quad Y_{n-2,1} \cdot x \psi
$$

and an element of $\operatorname{Im} \operatorname{Inf}_{L \cap \Gamma_{n-1}^{\prime}}^{\left(L \cap \Gamma_{n-1}^{\prime}\right) / Z}$; since $\phi, \psi$ belong to $\operatorname{Im} \operatorname{Inf}_{L \cap \Gamma_{n-1}^{\prime}}^{\left(L \cap \Gamma_{n-1}^{\prime}\right) / Z}$, by Lemmas 13 and 14, it follows that

$$
\operatorname{Res}_{K}^{L} \operatorname{tr}_{L}^{L \cap \Gamma_{n-1}^{\prime}} \operatorname{Res}_{L \cap \Gamma_{n-1}^{\prime}}^{\Gamma_{n-1}^{\prime}}\left(Y_{n-1,2}\right)=0,
$$

so $\operatorname{Res}_{K}^{\mathbb{E}_{n}}\left(\kappa_{n, 1}\right)=0$.

Finally, the case $\alpha=x_{2 n}$ follows from Lemma 19. The proposition is proved.

\section{ACKNOWLEDGMENTS}

Most of the results of this paper were obtained during a stay at the University of Essen and the ETH-Zentrum in Autumn 1997. I would like to thank Eckart Viehweg, Hélène Esnault and Urs Stammbach for making the visits possible. Many thanks to David John Green for valuable comments.

\section{REFERENCES}

1. A. Adem, D. Karagueuzian, Essential cohomology of finite groups, Comment. Math. Helv. 72 (1997), 101-109. MR 98f:20038

2. D.J. Benson, J.F. Carlson, The cohomology of extra-special groups, Bull. London Math. Soc. 24 (1992), 209-235; 25 (1993), 498. MR 93b:20087; MR 94f:20099

3. J. Carlson, Depth and transfer map in the cohomology of groups, Math. Z. 218 (1995), 461468. MR 95m:20058

4. T. Diethelm, The mod $p$ cohomology rings of the nonabelian split metacyclic p-groups, Arch. Math. (Basel) 44 (1985), 29-38. MR 86e:20057

5. J. Duflot, Depth and equivariant cohomology, Comment. Math. Helv. 56 (1981), 627-637. MR 83g:57029

6. D.J. Green, Private communication.

7. G. Hochschild and J. P. Serre, Cohomology of group extensions, Trans. Amer. Math. Soc. 74 (1953), 110-143. MR 14:619b

8. I.J. Leary, The mod $p$ cohomology rings of some p-groups, Math. Proc. Camb. Phil. Soc. 112 (1992), 63-75. MR 94a:20087

9. R.J. Milgram, M. Tezuka, The geometry and cohomology of $M_{12}$ : II, Bol. Soc. Mat. Mex. 1 (1995), 91-108. MR 97f:20064

10. P.A. Minh, H. Mui, The mod $p$ cohomology algebra of the group $M\left(p^{n}\right)$, Acta Math. Vietnamica 7 (1982), 17-26. MR 85i:20051

11. P.A. Minh, Hochschild-Serre spectral sequences, modular invariant theory and cohomology algebras of extra-special p-groups, Thesis, Univ. of Hanoi (1990).

12. 139-154. MR 93m:20074

13. The mod $p$ cohomology group of extra-special p-group of order $p^{5}$ and of exponent $p^{2}$, Math. Proc. Camb. Phil. Soc. 120 (1996), 423-440. MR 97b:20076

14. _ Transfer map and Hochschild-Serre spectral sequences, J. Pure Appl. Alg. 104 (1995), 89-95. MR 96h:20099

15. - On a conjecture of Kahn for Stiefel-Whitney classes of a regular representation, J. Alg. 188 (1997), 590-609. MR 98f:20039

16. H. Mui, The mod $p$ cohomology algebra of the group $E\left(p^{3}\right)$, Unpublished essay.

17. D. Quillen, The mod 2 cohomology rings of extra-special 2-groups and the spinor groups, Math. Ann. 194 (1971), 197-212. [MR 44:7582]

18. M. Tezuka, N. Yagita, The varieties of the mod $p$ cohomology rings of extra-special p-groups for an odd prime p, Math. Proc. Camb. Phil. Soc. 94 (1983), 449-459. MR 85g:20069 
19. Calculations in mod $p$ cohomology of extra special p-groups I, Contemp. Math. 158 (1994), 281-306. MR 95b:20074

20. E. Weiss, Cohomology of groups, Academic Press, New York, 1969. MR 41:8499

Department of Mathematics, College of Sciences, University of Hue, Dai hoc Khoa hoc, Hue, Vietnam

E-mail address: paminh@dng.vnn.vn

Current address: 53 Craig Road, Stockport SK4 2AP, England

E-mail address: minhp@vol.vnn.vn 